\title{
A bioactive phlebovirus-like envelope protein in a hookworm
} endogenous virus

3 Monique Merchant ${ }^{1,2, \dagger}$, Carlos P. Mata ${ }^{1,2, \dagger, \ddagger}$, Yangci Liu ${ }^{1,2}$, Haoming Zhai ${ }^{1,2}$, Anna V. Protasio ${ }^{3,4}$, Yorgo Modis ${ }^{1,2 *}$

$5{ }^{1}$ Molecular Immunity Unit, Department of Medicine, University of Cambridge, MRC Laboratory of

6 Molecular Biology, Francis Crick Avenue, Cambridge, CB2 0QH, United Kingdom

$7 \quad{ }^{2}$ Cambridge Institute of Therapeutic Immunology \& Infectious Disease (CITIID), University of

8 Cambridge School of Clinical Medicine, Cambridge CB2 OAW, United Kingdom

$9{ }^{3}$ Department of Pathology, University of Cambridge, Tennis Court Road, Cambridge CB2 1QP,

10 United Kingdom

$11{ }^{4}$ Christ's College, University of Cambridge, St Andrew's Street, Cambridge, CB2 3BU, United

12 Kingdom

13 TThese authors contributed equally to this work

14 †Present address: Electron and Confocal Microscopy Unit, National Centre for Microbiology,

15 28220, Majadahonda, Madrid, Spain

$16{ }^{*}$ Corresponding author: ymodis@mrc-Imb.cam.ac.uk

18 Keywords

19 Transposable element; retrotransposon; endogenous retrovirus (ERV); envelope protein;

20 membrane fusion; horizontal gene transfer (HGT); virus evolution; gene exaptation; nematode

\section{Short title (50 characters)}

23 A bioactive phlebovirus-like Env in a hookworm EVE 


\section{Abstract}

25 Endogenous viral elements (EVEs), accounting for $15 \%$ of our genome, serve as a genetic

26 reservoir from which new genes can emerge. Nematode EVEs are particularly diverse and

27 informative of virus evolution. We identify Atlas virus - an intact retrovirus-like EVE in the

28 human hookworm Ancylostoma ceylanicum, with an envelope protein genetically related to GN-

29 GC glycoproteins from phleboviruses. A cryo-EM structure of Atlas GC reveals a class II viral

30 membrane fusion protein fold not previously seen in retroviruses. Atlas GC has the structural

31 hallmarks of an active fusogen. Atlas GC trimers insert into membranes with endosomal lipid

32 compositions and low $\mathrm{pH}$. When expressed on the plasma membrane, Atlas GC has cell-cell

33 fusion activity. RNA-Seq data analysis detected transcripts mapping to Atlas virus at different

34 stages of hookworm development. With its preserved biological activities, Atlas GC has the

35 potential to acquire a cellular function. Our work reveals structural plasticity in reverse-

36 transcribing RNA viruses.

\section{Introduction}

39 Retroviruses and other reverse-transcribing RNA viruses can frequently integrate their genome,

40 reverse-transcribed from RNA into DNA, into the host-cell genome. Viral genomes integrated

41 into germline cells are inherited by future generations as endogenous viral elements (EVEs).

42 Most EVEs are endogenous retroviruses (ERVs) and account for approximately $15 \%$ of the

43 human genome, seven times more than protein-coding genes (1, 2). EVEs and other

44 transposons were initially viewed as parasitic DNA. It is now evident that they serve as a genetic

45 reservoir, from which new genes and regulatory elements can emerge. Sequences of retroviral

46 origin help control gene expression by serving as promoters, enhancers and other regulatory

47 elements $(3,4)$. Genes coopted from EVEs have evolved to fulfill vital cellular functions (1). For

48 example, syncytins, which drive cell-cell fusion of trophoblasts during placental development,

49 are encoded by ERV envelope glycoprotein (env) genes $(3,5)$. Another recent example is the 
50 Gag capsid protein encoded by the Caenorhabditis elegans Cer1 retrotransposon, which

51 encapsidates small non-coding RNAs into nucleocapsids that can transfer RNAs conferring

52 learned pathogen avoidance behavior from infected parents to naïve progeny (6).

53 The reduced mutation rate of host versus retrovirus genomes $\left(10^{-9}\right.$ vs. $10^{-3}$ mutations

54 per site per year (7)) means that EVEs are windows to ancestral retroviral sequences -

55 evolutionary fossils preserved from the time of integration (2). Some EVE genes are expressed

56 in human tissues and retain their biological activities, such as membrane fusion activity in the

57 case of Env proteins (Envs) (3, 5, 8). Aberrant expression of Envs is associated with disease (9,

58 10). With the biology of EVEs still largely uncharted, it is likely that many cellular functions of

59 EVEs in health and disease remain undiscovered. Studying EVE genes with novel properties

60 could therefore provide insights on the evolutionary history of reverse-transcribing RNA viruses

61 and identify fundamental principles in host-virus coevolution.

Nematode EVEs are particularly diverse and informative of virus evolution. EVEs from

63 the Belpaoviridae (BEL/Pao) family (11), related to retroviruses and widespread across

64 metazoa, have revealing genetic features in nematodes (12). The presence in C. elegans EVEs

65 of overlapping ORFs, otherwise unique to complex vertebrate retroviruses, suggests

66 retroviruses originated in early metazoa with a common ancestor resembling belpaoviruses

67 (13). Furthermore, nematode endogenous belpaoviruses encode Envs that are genetically

68 unrelated to retrovirus Envs (14). Instead of a class I viral membrane fusion protein with a core

69 fold of three bundled a-helices (15-18) - a defining feature of modern retroviruses (19) -

70 belpaovirus Envs have sequence similarity to $G_{C}(G 2)$ envelope glycoproteins from

71 phleboviruses (family Phenuiviridae) (14). $\mathrm{G}_{c}$ proteins are class II membrane fusion proteins,

72 with a three-domain $\beta$-strand architecture $(20,21)$ also found in alphaviruses (22), flaviviruses

$73(23,24)$ and Rubella virus $(25)$ but structurally unrelated to class I fusion proteins. A series of

74 conformational changes in class II fusion proteins, triggered by endosomal acidification, 
75 catalyses fusion of the viral and endosomal membranes to deliver the viral genome into the

76 cytosol (26-28). A hydrophobic fusion loop first inserts into the endosomal membrane. The

77 proteins then form trimers and fold back on themselves, pulling the cell membrane (held by the

78 fusion loop) and the viral membrane (held by a transmembrane anchor) together so they fuse $79(19,21)$.

Class II fusion proteins are not limited to viruses: they also drive cell-cell fusion events of

81 fundamental importance, including syncytial epithelia formation in C. elegans and other

82 nematodes (29-32), and gamete fusion in protozoa, plants, algae and invertebrates (33-38). The

83 identical topology and overall arrangement of the three domains of viral and eukaryotic class II

84 fusion proteins, along with similarities in their membrane fusion mechanisms, makes it all but

85 certain they evolved from a common ancestor (33). Although the evolutionary origin of the

86 ancestral class II fusion protein remains unknown, the presence of class II fusion proteins in

87 EVEs raises the provocative prospect that a gene transfer from a virus to a cell led to the advent

88 of sexual reproduction $(33,39)$.

Here, we identify a novel, intact endogenous belpaovirus in the human hookworm

90 Ancylostoma ceylanicum (a parasitic nematode) with an Env more similar than any other

91 eukaryotic sequence to phlebovirus $G_{c}$ protein sequences. We expressed and purified the $G_{C^{-}}$

92 homologous fragment from this EVE, henceforth Atlas virus. A cryo-electron microscopy (cryo-

93 EM) structure of Atlas $G_{C}$ reveals a class II viral fusion protein fold remarkably similar to

94 phlebovirus $G_{c}$ proteins and not seen in retroviruses, as predicted 20 years ago $(12,14)$. We

95 show that Atlas $G_{C}$ has all the hallmarks of an active class II membrane fusion protein. It

96 undergoes a monomer-to-trimer transition and inserts into lipid membranes with a specific lipid

97 composition in response to a low $\mathrm{pH}$ trigger. Our work provides biochemical validation for the

98 hypothesis that acquisition of a fusion protein from an infectious virus, as exemplified by Atlas

99 virus, represents a general paradigm for how retrotransposons can become retroviruses (14), 
100 and indeed how ancestral reverse-transcribing viruses may have originated (11). The preserved

101 biological activities of Atlas $\mathrm{G}_{\mathrm{c}}$, including membrane fusion activity, raise the question of

102 whether these activities, and those of EVE gene products more broadly, have cellular functions

103 or cause disease.

\section{Results}

\section{An intact EVE with a phlebovirus-like Env in the hookworm A. ceylanicum}

106 The bioinformatic discovery of nematode EVEs with phlebovirus $\mathrm{G}_{c}$-like Env sequences not 107 seen in retroviruses $(12,14)$, requires biochemical validation. To identify phlebovirus-like EVE

108 Envs suitable for biochemical analysis, we performed PSI-BLAST searches (40) for protein

109 sequences similar to biochemically characterized phlebovirus $G_{c}$ proteins. A search with Rift

110 Valley Fever virus (RVFV) Gc as the query identified the gene Acey_s0020.g108 (UniProt

111 A0A016UZK2) in the human hookworm $A$. ceylanicum as containing the most similar sequence

112 outside infectious virus taxa (Expected value $10^{-20}$ ). The homologous sequence lies within a

$1139,204-$-nucleotide element possessing all the features of an intact EVE, including 100\% identical

114 271-nt LTRs and a coding sequence encoding a single 2,828-residue Gag-Pol-Env polyprotein

115 without any stop codons or introns (Fig. 1A and fig. S1A). We refer to this element as $A$.

116 ceylanicum Atlas virus. It is one of nine A. ceylanicum EVEs that encode complete Gag-Pol-Env

117 polyproteins (Fig. 1, B and C). These EVEs have the distinguishing genomic features of

118 belpaoviruses from other nematode species, including an atypical Env and an aspartate to

119 asparagine substitution $(\mathrm{Y}[\mathrm{X}] \mathrm{DD} \rightarrow \mathrm{YVDN})$ in the most conserved reverse transcriptase motif,

120 the polymerase site $(13,41,42)$ (fig. S1B). Phylogenetic classification of Atlas virus based on

121 reverse-transcriptase (RT) sequences confirms the Belpaoviridae phylogeny of Atlas virus, with

122 Cer13 from C. elegans (12) as the closest neighbor in the RT tree (Fig. 1D). With a Gag

123 sequence less than $20 \%$ identical to its closest homolog (Acey_s0020.g1106), the Atlas EVE is 
124 a candidate for classification as a member of the Belpaoviridae family (which contains a single

125 genus, Semotivirus) (fig. S1C) (43).

126 The phlebovirus $\mathrm{G}_{\mathrm{C}}$-like sequence spans the last 498 residues of the Atlas virus

127 polyprotein (residues 2330-2828). It contains a single predicted C-terminal transmembrane

128 helix, like phlebovirus $\mathrm{G}_{\mathrm{c}}$ proteins. Phleboviruses and other Phenuiviridae family members

129 express a glycoprotein precursor that is cleaved by cellular proteases into two envelope

130 glycoproteins, $G_{N}$ and $G_{C}$ (or $G 1$ and $G 2$ ). $G_{N}$ is the receptor-binding protein required for cellular

131 attachment and $G_{C}$ is the membrane fusion protein required for cell entry. $G_{N}$ is highly antigenic

132 and more variable in sequence than $\mathrm{G}_{\mathrm{c}}$. Our analysis of the Atlas virus Env sequence detected

133 a slight but statistically significant similarity in the 421 residues preceding the $\mathrm{G}_{\mathrm{C}}$-like sequence

134 (residues 1909-2329) to $\mathrm{G}_{N}$ glycoproteins from Phenuiviridae family viruses $\left(E\right.$-value $\left.\geq 10^{-7}\right)$.

135 Moreover, the distribution of predicted transmembrane helices and proteolytic cleavage sites in

136 and adjacent to the Atlas $G_{N^{-}}$and $G_{C}$-like sequences is the same as in phlebovirus

137 glycoproteins (Fig. 1A). Together, these sequence features suggest the Atlas virus Env

138 contains tandem phlebovirus-like $G_{N}$ and $G_{C}$ glycoproteins instead of a retrovirus-like

139 glycoprotein. With all the features of a recently active EVE and an apparently intact set of

140 phlebovirus-like glycoproteins, Atlas virus is an excellent candidate for biochemical analysis. 


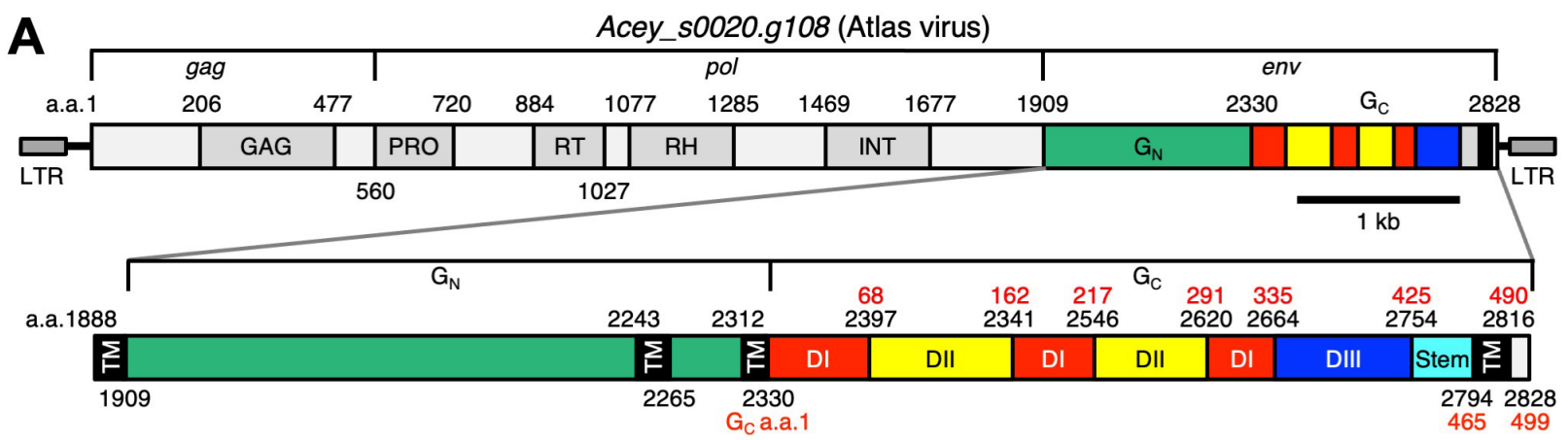

B

C

\begin{tabular}{|c|c|c|c|c|c|}
\hline ERV gene name & Strand & $\begin{array}{l}\text { LTR } \\
\text { (bp) }\end{array}$ & $\begin{array}{c}\text { LTR } \\
\text { (\%ID) }\end{array}$ & $\begin{array}{c}\text { \%aa s } \\
\text { Env }\end{array}$ & $\begin{array}{r}\text { eq ID } \\
\text { Gag }\end{array}$ \\
\hline Acey_s0020.g108 & - & 271 & 100 & 100 & 100 \\
\hline Acey_s0009.g673 & + & 350 & 100 & 20 & 12 \\
\hline Acey_s0023.g802 & + & 219 & 98.2 & 16 & 16 \\
\hline Acey_s0023.g869 & + & 356 & 100 & 18 & 12 \\
\hline Acey_s0025.g1106 & + & $<100$ & - & 21 & 19 \\
\hline Acey_s0070.g451 & + & 382 & 99.5 & 19 & 12 \\
\hline Acey_s0203.g1846 & - & 499 & 99.8 & 18 & 15 \\
\hline Acey_s0206.g1994 & + & $<100$ & - & 17 & 12 \\
\hline Acey_s0398.g719 & + & 207 & 100 & 10 & 12 \\
\hline
\end{tabular}
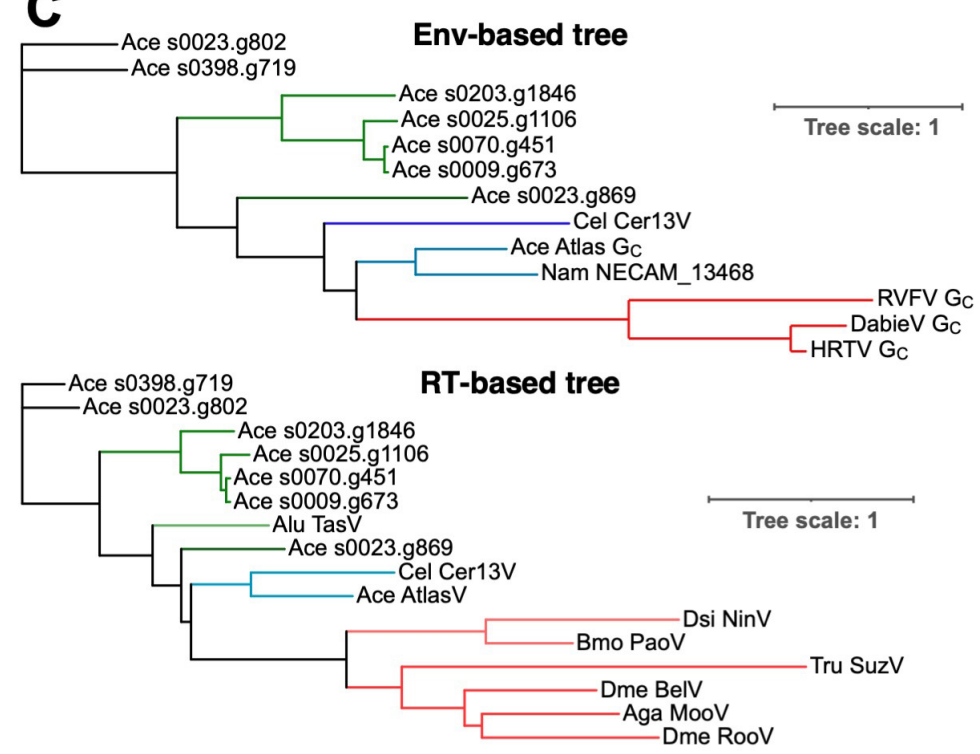

143 Fig. 1. Atlas is an endogenous belpaovirus with a phlebovirus-like Env. (A) Gene

144 architecture of the Atlas endogenous viral element (EVE), gene Acey_s0020.g108 from the

145 Ancylostoma ceylanicum human hookworm parasite. Inset: annotated closeup of the Atlas Env

146 region encoding phlebovirus $\mathrm{G}_{N^{-}}$and $\mathrm{G}_{\mathrm{C}}$-like glycoproteins. Red residue number refer to the $\mathrm{G}_{\mathrm{C}}$

147 sequence alone. (B) List of $A$. ceylanicum EVEs encoding complete Gag-Pol-Env polyproteins

148 with phlebovirus-like Envs. LTR sequence identities for two of the EVEs could not be calculated:

149 Acey_s0206.g1994 had degenerate LTRs, and one LTR of Acey_s0025.g1106 was truncated in

150 the genome assembly. (C) Phylogenetic tree of $\mathrm{G}_{\mathrm{C}}$ proteins from Phenuiviridae and $\mathrm{G}_{\mathrm{C}}$-like (G2-

151 like) sequences from EVEs. Ace, A. ceylanicum; Cel, C. elegans; Nam, Necator Americanus,

152 RVFV, Rift Valley Fever virus; DabieV, Dabie bandavirus (SFTSV); HRTV, Heartland virus. (D)

153 Phylogenetic classification of Atlas based on reverse-transcriptase (RT) sequences. Alu Tas V, 
154 Ascaris lumbricoides Tas virus; Dsi NinV, Drosophila simulans Ninja virus; Bmo PaoV, Bombyx

155 mori Pao virus; Tru SuzV, Takifugu rubripes Suzu virus; Dme BelV, D. melanogaster Bel virus;

156 Aga MooV, Anopheles gambiae Moose virus; RooV, Roo virus. Trees drawn with iTOL v6 (44).

157 Atlas $\mathbf{G}_{\mathrm{c}}$ has a class II membrane fusion protein fold not seen in retroviruses

158 As the molecular structure of phlebovirus $\mathrm{G}_{\mathrm{C}}$ proteins, and how they drive fusion of the viral and

159 cellular membranes, are well established from previous studies $(20,28)$, we focused our

160 biochemical analyses on the $\mathrm{G}_{\mathrm{c}}$-like sequence from Atlas virus. A recombinant ectodomain

161 fragment of Atlas $\mathrm{G}_{\mathrm{C}}$ (polyprotein residues 2330-2772) was expressed in Drosophila

162 melanogaster D.mel-2 cells as a secreted protein. The purified protein was a soluble, folded

163 homotrimer (fig. S2). The structure of the $\mathrm{G}_{\mathrm{C}}$ trimer was determined by single-particle cryo-EM

164 image reconstruction at an overall resolution of $3.76 \AA$ (Fig. 2, A and B; table S1; fig. S3). The

165 map was sufficiently detailed for an atomic model to be built and refined for Atlas $\mathrm{G}_{\mathrm{c}}$ residues

166 2330-2769 using the crystal structure of RVFV G $(28)$ as a starting model (see Methods; fig.

167 S4). The structure reveals a three-domain class II membrane fusion protein fold (Fig. 2C). All

168 previously described retroviral Env structures have a helical coiled-coil class I fusion protein fold

169 (15-18), exemplified by the hemagglutinin of influenza virus (19). The structure of the Atlas $\mathrm{G}_{c}$

170 ectodomain fragment is remarkably similar to phlebovirus $\mathrm{G}_{\mathrm{C}}$ structures, specifically the trimeric

171 postfusion $\mathrm{G}_{c}$ structures from RVFV (28), Dabie bandavirus (DABV, formerly SFTS phlebovirus)

172 (45) and Heartland virus (HRTV) (46) (Fig. 2, D and E). Domain I, a 10-stranded $\beta$-barrel

173 augmented by a 3-stranded sheet, organizes the structure. Two insertions in domain I form the

174 elongated, mostly $\beta$-stranded domain II. Domain III has the 7-stranded $\beta$-sandwich topology of

175 fibronectin type III (FN3) domains also found in macroglobulin domains $(35,47)$, but the

176 hydrophobic core and disulfide bonding pattern of domain III differ from these and other

177 annotated domains from non-viral species. A 16-amino acid portion of the stem region, which

178 links domain III to the C-terminal transmembrane anchor in class II fusion proteins, could be 
179 modeled, spanning $5 \mathrm{~nm}$ from the end of domain III to within approximately $1 \mathrm{~nm}$ of the tip of

180 domain II. The stem forms trimer contacts, adding a $\beta$-strand to domain II of a different subunit,

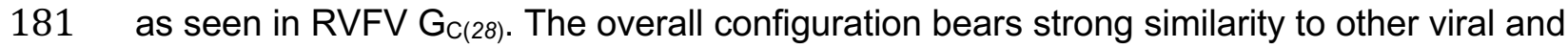

182 cellular class II fusion proteins including, in order of decreasing similarity: alphavirus E1 proteins

183 (22); EFF-1/AFF-1 cell-cell fusion proteins from C. elegans and other animals (29, 30); HAP2

184 gamete fusion proteins from protozoa $(33-35,48)$ and plants $(34,36)$; and flavivirus E proteins

185 (49) (Fig. 2E). Despite these structural similarities, the only proteins or domains of known

186 structure with detectable amino acid sequence similarity to Atlas $\mathrm{G}_{\mathrm{C}}$ ( $E$-value $<1$ in PSI-BLAST

187 (40)) are the phlebovirus $\mathrm{G}_{c}$ proteins (22-24\% sequence identity; fig. S5).

Atlas $G_{C}$ has the same structural features that distinguish phlebovirus glycoproteins from

189 other class II fusion proteins: a larger number of disulfide bonds, ten of which are conserved in

190 phlebovirus $\mathrm{G}_{c}$ sequences but not in other class II proteins; N-linked glycosylation in domain III;

191 and a more extensive and rigid interface between domains I and II (Fig. 2, D and E, and fig.

192 S5). The most notable differences between Atlas virus and phlebovirus $\mathrm{G}_{c}$ structures are

193 differences in the disulfide bonding pattern and in the composition of side chains lining the

194 glycerophospholipid (GPL) headgroup binding pocket conserved in arboviral class II fusion

195 proteins (28). We discuss these differences and their potential functional implications below.

196 Atlas $\mathrm{G}_{\mathrm{C}}$ also has a different glycosylation pattern, with a single predicted $\mathrm{N}$-linked glycosylation

197 site at Asn414 in domain III with a weak corresponding feature in the density map (fig. S6). In

198 contrast, phlebovirus $\mathrm{G}_{c}$ proteins contain two N-linked glycans in domain III, at two different

199 sites. One of these, Asn1035 in RVFV, covers the fusion loop in the prefusion conformation of

200 RVFV $G_{C}$ and stabilizes the prefusion dimer by forming contacts across the dimer interface (20),

201 as also seen in flavivirus E proteins $(23,24)$. This glycosylation site is conserved in DABV and

202 HRTV $G_{c}$ but absent in Atlas $G_{c}$. Despite these minor differences, the striking overall structural

203 similarity of Atlas $G_{c}$ to phlebovirus $G_{c}$ proteins in the postfusion conformation experimentally 
204 validates the evolutionary link between nematode EVEs from the Belpaoviridae family and the

205 fusion proteins of phleboviruses postulated based on previous genomic analyses (12, 14).

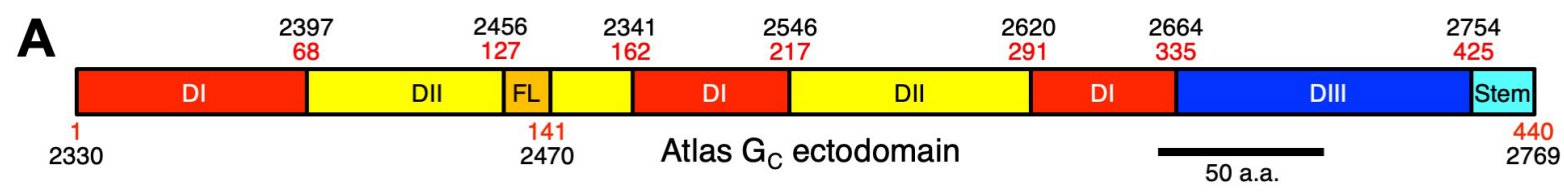
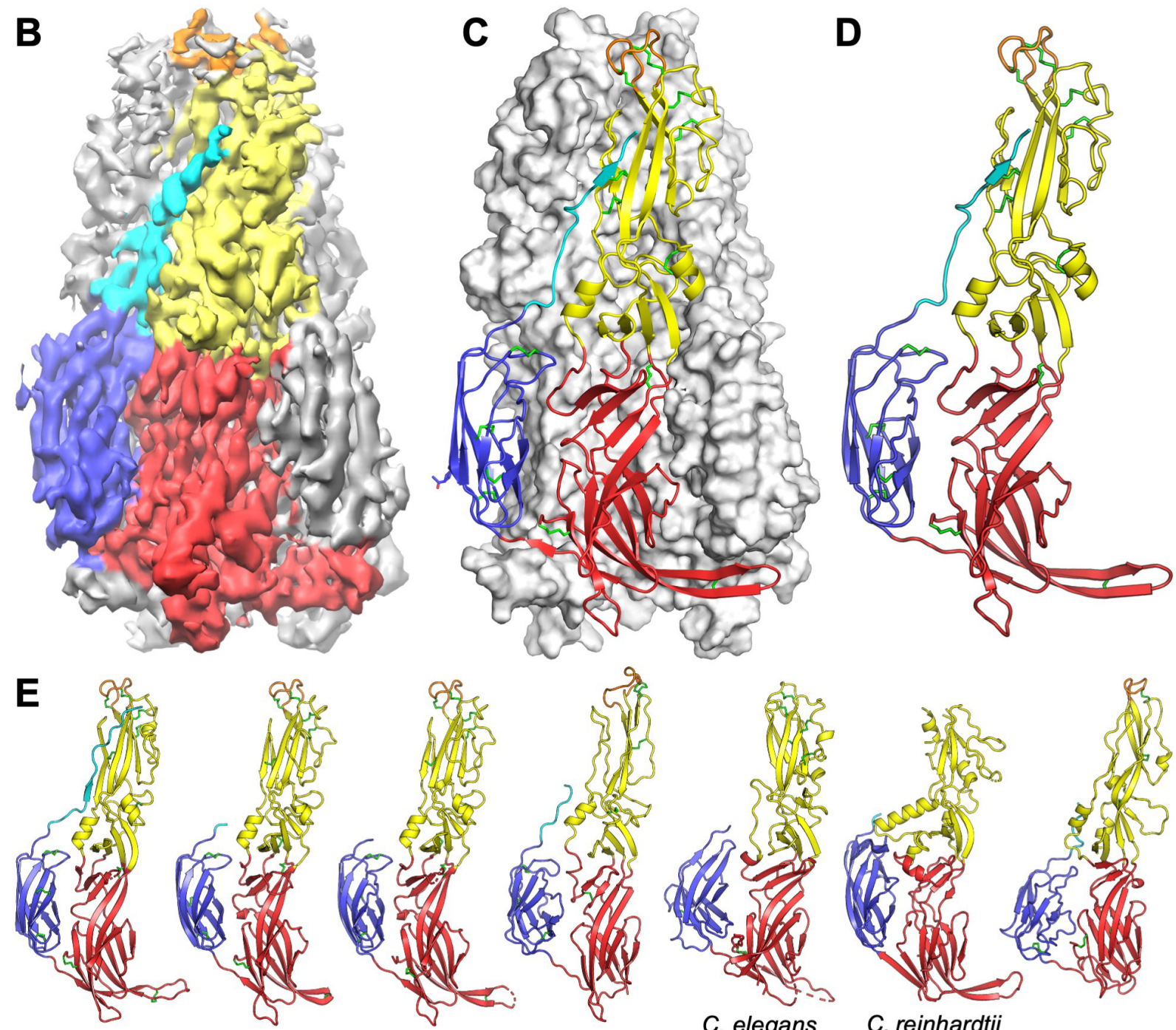

RVFV $G_{C}$

DABV G

HRTV $G_{C}$

SFV E1
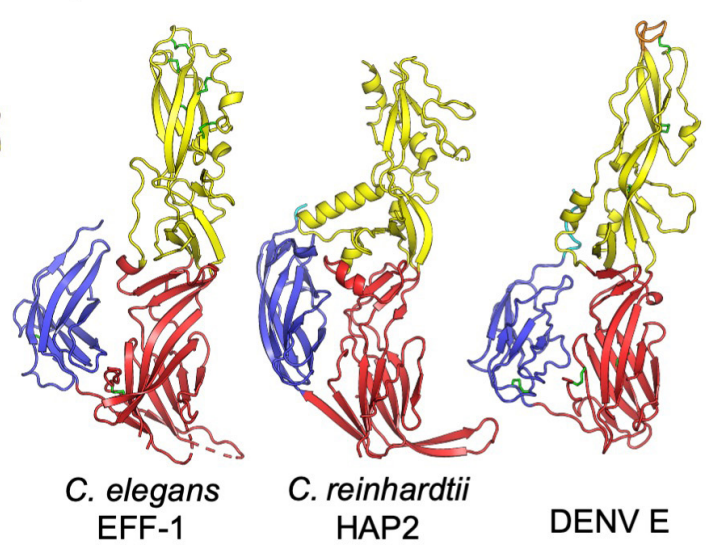

207 Fig. 2. Cryo-EM structure of a phlebovirus $\mathbf{G}_{c}$-like Env fragment from Atlas virus. (A)

208 Domain organization of Atlas Gc. (B) Cryo-EM image reconstruction of a soluble ectodomain

209 fragment of Atlas $G_{c}$ at $3.76 \AA$ overall resolution. The purified protein was a homotrimer (fig. S2)

210 and threefold (C3) symmetry was imposed. The map is colored by domain as in (A). A

211 representative cryo-EM micrograph is shown in fig. S4A. (C) Overview of the refined atomic 
212 model of the Atlas $\mathrm{G}_{\mathrm{c}}$ trimer. Disulfide bonds (green) and an N-linked glycan (blue) are shown

213 as sticks. (D) Protein fold of an Atlas $G_{c}$ protomer. (E) The structures most similar to Atlas $G_{c}$

214 based on structure comparison with DALI (50), $\mathrm{G}_{\mathrm{C}}$ glycoproteins from Rift Valley Fever virus

215 (RVFV, Rmsd 2.6 A, Z-score 29, PDB:6EGU (28)), Dabie bandavirus (DABV, formerly SFTS

216 phlebovirus; Rmsd 2.6 A, Z-score 28, PDB:5G47 (45)) and Heartland virus (HRTV, Rmsd $2.6 \AA$,

217 Z-score 28, PDB:5YOW (46)). Other representative class II fusion proteins are shown for

218 comparison: Semliki Forest virus (SFV) E1 (Z-score 19, PDB:1RER (27)), C. elegans EFF-1 (Z-

219 score 19, PDB:4OJD (29)), Chlamydomonas reinhardtii HAP2 (Z-score 16; PDB:5MF1 (33)) and

220 dengue virus (DENV) E (Z-score 15, PDB:4GSX (51)).

\section{Structure of the putative lipid membrane anchor of Atlas $\mathbf{G}_{\mathrm{c}}$}

222 Viral fusion proteins insert a membrane anchor - the fusion loop, in class II proteins - into the

223 host cell membrane to initiate virus-cell membrane fusion. The putative fusion loop of Atlas $\mathrm{G}_{\mathrm{C}}$

224 can be identified by analogy to phlebovirus $\mathrm{G}_{c}$ proteins as spanning residues $127-140$. The local

225 resolution of the cryo-EM density for this region is lower than for the rest of the map but the

226 large number of structural constraints imposed by the positions of disulfide-bonded cysteines

227 and other residues conserved in phleboviruses $G_{c}$ proteins allowed an atomic model to be built

228 unambiguously (Fig. 3A and fig. S6, B and C). Specifically, the fusion loop is constrained by

229 four disulfide bonds conserved in phleboviruses, a fifth disulfide specific to Atlas $\mathrm{G}_{c}$ (Cys129-

230 Cys138), a phenylalanine (Phe136) required in phleboviruses at the apex of the fusion loop for

231 membrane binding and fusion (28, 45, 52), and two conserved glycines (Gly128 and Gly134)

232 that provide the torsional flexibility necessary for the fusion loop's tightly folded conformation

233 (Fig. 3, A to C).

234 The structure and chemical properties of the Atlas $\mathrm{G}_{\mathrm{C}}$ fusion loop resemble phlebovirus

235 fusion loops. Ile130, Phe136, Phe137 and the Atlas-specific disulfide (Cys129-Cys138) form a

236 hydrophobic surface similar to phlebovirus and flavivirus fusion proteins $(26,28)($ Fig. 3 C). The 
237 area of this surface is greater than in most viral class II fusion proteins. By analogy with other

238 viral class II fusion proteins, the location and extent of the hydrophobic surface formed by the

239 Atlas $\mathrm{G}_{\mathrm{c}}$ fusion loop suggest it could function as a membrane anchor.

240 In addition to inserting nonpolar side chains into the hydrophobic region of the

241 membrane, viral class II fusion proteins form polar contacts with lipid headgroups via the fusion

242 loop and an adjacent glycerophospholipid (GPL) binding pocket (28). By selecting for

243 headgroups with complementary electrostatic potential, polar contacts confer a degree of

244 specificity to lipid binding. In phleboviruses, a set of conserved polar residues in the GPL

245 binding pocket bind selectively to zwitterionic GPLs (28). The Atlas $\mathrm{G}_{\mathrm{c}}$ structure reveals a

246 putative GPL binding pocket with both conserved and novel features (Fig. 3). The arginine that

247 forms bidentate hydrogen bonds with the GPL phosphate moiety in phleboviruses (28) is

248 conserved in Atlas virus (Arg86). The disulfide bond and short-chain hydrophobic residue that

249 bind the GPL glycerol moiety are also conserved (Cys87-Cys135, Ala90). However, an

250 aspartate-arginine pair that binds choline and ethanolamine GPL moieties in phleboviruses is

251 replaced in Atlas $G_{c}$ by two histidines (His271 and His85). Moreover, Atlas virus has an extra

252 residue in the fusion loop, Asp133, compared to phlebovirus $G_{c}$ proteins. The Asp133 side

253 chain points into the putative GPL binding pocket and is located near the position of the choline

254 GPL moiety in the superimposed structure of RVFV $\mathrm{G}_{c}$ bound to a phosphatidylcholine ligand

255 (28), suggesting that Asp133 could compensate for the lack of a conserved aspartate at position

256271 (Fig. 3, A to C). Hence the putative GPL binding pocket of Atlas $\mathrm{G}_{c}$ appears to have the

257 necessary physicochemical attributes to support GPL binding, with Arg86 binding the phosphate

258 moiety, Cys87/Cys135/Ala90 binding the glycerol moiety, and His85/Asp133/His271

259 coordinating the end of the headgroup. We note the presence in the cryo-EM reconstruction of a

260 bulge in the density around the GPL binding pocket that is unaccounted for by the atomic model

261 (fig. S6C). Additionally, the absorbance at $260 \mathrm{~nm}$ of purified Atlas $\mathrm{G}_{\mathrm{c}}$ was higher than 
262 expected despite treatment with nucleases during purification (fig. S2A). These two

263 observations would be consistent with lipid molecules with unsaturated acyl chains copurifying

264 with Atlas $\mathrm{G}_{\mathrm{c}}$, but the local resolution of the map is insufficient to ascertain whether the GPL

265 binding pocket contains a ligand.

A

A Atlas HRCHRAGSCHSDECHHVKDTDALPEFSSEANSRP-GYTSCSSSCGCITCDGCFFCEPSCLF

$.|||\ldots| .|| \ldots . \ldots \ldots|\ldots \ldots||| \ldots|\ldots \ldots| \ldots|| \ldots|||| \ldots|||| \mid$ RVFV RRCHLVGECHVNRCLSWRDNETSAEFSFVGESTTMRENKCFEQCGGWGC-GCFNVNPSCLF

C

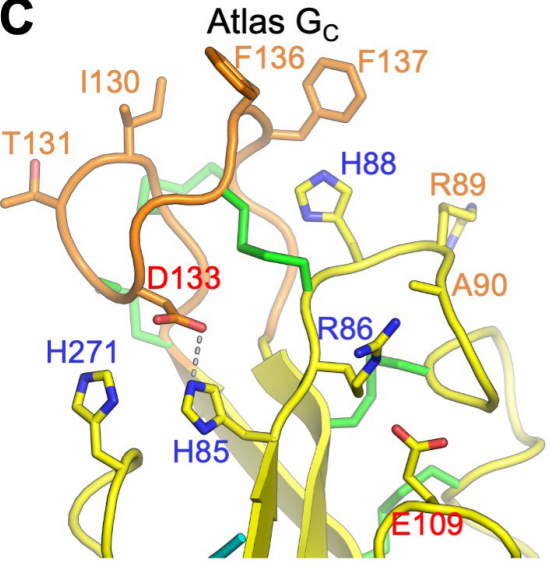

D

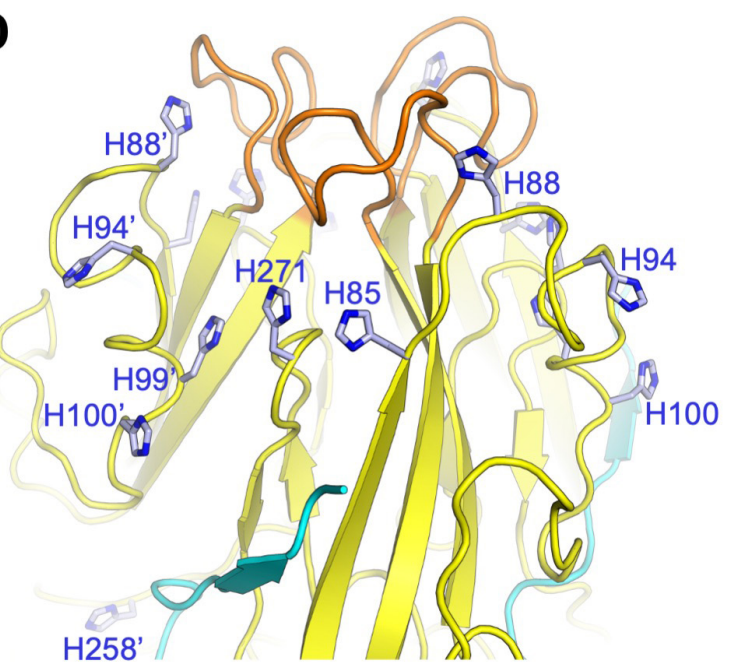

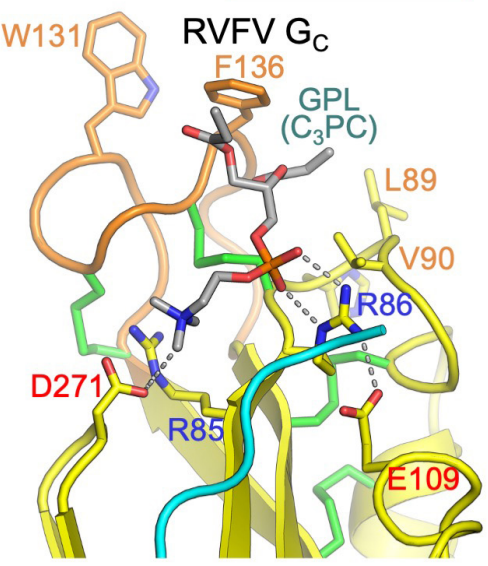

$\mathbf{E}$
B GPL pocket residues
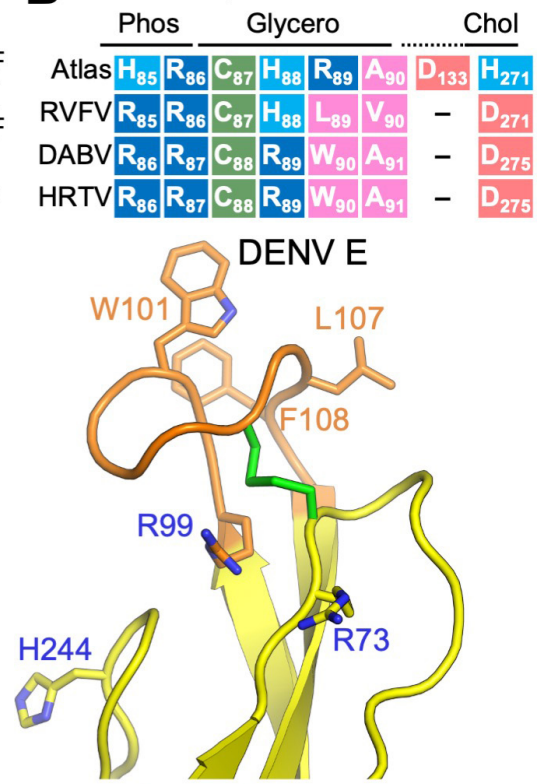

Fig. 3. Structure of the putative fusion loop and lipid binding pocket of Atlas $\mathbf{G}_{c \text {. }}(A)$ Local sequence alignment of the fusion loops (orange boxes) of Atlas $\mathrm{G}_{\mathrm{c}}, \mathrm{RVFV} \mathrm{G}$, and DENV E.

269 Green dumbbells indicate disulfides. Disulfides shown below the RVFV sequence are conserved in Atlas and RVFV. (B) Residues contributing to binding of the phosphate (Phos), 
271 glycerol and choline (Chol) moieties of glycerophospholipid (GPL) molecules by RVFV G $(28)$.

272 Colors indicate side chain properties: blue, positive charge; pink, negative charge; magenta,

273 hydrophobic; green, sulfhydryl; light blue, positive charge at endosomal $\mathrm{pH}$. (C) Closeup of the

274 fusion loop and GPL binding pockets of Atlas $\mathrm{G}_{c}, \mathrm{RVFV} \mathrm{G}$ and dengue E. A single protomer

275 from the postfusion trimer is shown, with key residues shown in stick representation. Label

276 colors indicate side chain properties: blue, positive charge; red, negative charge; orange,

277 hydrophobic. (D) Closeup of the fusion loop and GPL binding pocket of the Atlas $\mathrm{G}_{c}$ trimer

278 showing histidine residues in or near the pocket. Prime symbols following residue numbers

279 denote the protomer to which the residue belongs. (E) Surface representation of the same view

280 as in (D), colored by side chain hydrophobicity, except for histidine residues shown in light blue.

\section{Atlas $\mathrm{G}_{\mathrm{c}}$ binds membranes with endosome-like lipid composition at low $\mathrm{pH}$}

282 A key step in viral membrane fusion is binding of the fusion protein to the host cell membrane.

283 We assessed binding of Atlas $\mathrm{G}_{c}$ ectodomain to liposomes in density gradient centrifugation

284 and dynamic light scattering (DLS) experiments. Viruses containing class II fusion proteins, like

285 many retroviruses, undergo membrane insertion and fusion in endosomal compartments where

286 the $\mathrm{pH}$ is acidic (53-56). We therefore assayed liposome binding at a range of $\mathrm{pH}$ values. In

287 contrast to RVFV $G_{c}$ ectodomain, Atlas $G_{c}$ ectodomain did not bind liposomes containing

288 phosphatidylcholine (PC), phosphatidylethanolamine (PE), cholesterol and sphingomyelin (SM)

289 at neutral or acidic $\mathrm{pH}\left(\mathrm{pH}\right.$ 4-8; Fig. 4, A and B). At neutral $\mathrm{pH}(\mathrm{pH} 7.8)$, Atlas $\mathrm{G}_{\mathrm{c}}$ ectodomain

290 also failed to bind liposomes containing anionic lipids enriched in early or late endosomes:

291 phosphatidylserine (PS) or bis(monoacylglycerol)phosphate (BMP, also known as

292 lysobisphosphatidic acid or LBPA), respectively. At pH 4, however, Atlas $\mathrm{G}_{c}$ ectodomain bound

293 tightly to liposomes containing PS or BMP, with weaker binding observed at pH 4.6 (Fig. 4, A

294 and B, and fig. S7). Remarkably, Atlas $G_{c}$ ectodomain bound only weakly to liposomes

295 containing phosphatidylglycerol (PG) instead of BMP even though PG and BMP are 
296 regioisomers with identical chemical composition and electrostatic charge (of -1). PG and BMP

297 differ only in the position of the second acylglycerol linkage, resulting in a linear configuration for

298 BMP instead of the usual branched configuration for PG. Our liposome binding data show that

299 Atlas $\mathrm{G}_{\mathrm{c}}$ binds to membranes containing specific GPLs that are enriched in the endosomal

300 pathway in a pH-dependent manner. No other class II fusion protein has been reported to

301 require low pH, PS or BMP for membrane insertion. However, phleboviruses, which require only

302 PE or PC and cholesterol for membrane insertion (28), subsequently require BMP and low $\mathrm{pH}$

303 for fusion (57). Similarly, flaviviruses require BMP, PS or other anionic lipids and low $\mathrm{pH}$ for

304 efficient fusion $(53,55,58)$.

The optimal $\mathrm{pH}$ for membrane binding of Atlas $\mathrm{G}_{\mathrm{C}}(\mathrm{pH} 4-4.5)$ is similar to the optimal $\mathrm{pH}$

306 of hemifusion of Uukuniemi virus (57), a model phlebovirus, and would be consistent with

307 membrane insertion in late endosomes, as is the case for phleboviruses (54). The side chains of

308 His85 and His271, in the GPL binding pocket of Atlas $\mathrm{G}_{c}$, would be fully protonated at $\mathrm{pH}$ 4-4.5.

309 The resulting net positive charge of the His85/Asp133/His271 triad (+1/-1/+1), analogous to the

$310 \mathrm{Arg} / \mathrm{Asp}$ pair that coordinates the end of GPL headgroups in phleboviruses, mirrors the charge

311 of the phosphoserine headgroup of PS (-1/+1/-1). Moreover, Atlas $\mathrm{G}_{c}$ contains four additional

312 solvent-exposed histidines (residues $88,94,99$ and 100) in the vicinity of the GPL binding

313 pocket (Fig. 3, D and E). Protonation of these histidines at low pH may promote further

314 interactions with anionic lipid headgroups. The presence of six histidines in and around the GPL

315 binding pocket provides a possible explanation for the observed $\mathrm{pH}$-dependent insertion of Atlas

$316 \mathrm{G}_{\mathrm{c}}$ into membranes containing PS and BMP. Consistent with a conserved role for the GPL

317 binding pocket in determining lipid specificity of class II fusion proteins, mutations in

318 alphaviruses at a position equivalent to His271, in the $i j$ loop, determine the extent to which

319 alphaviruses depend on cholesterol for membrane binding (59). 
A

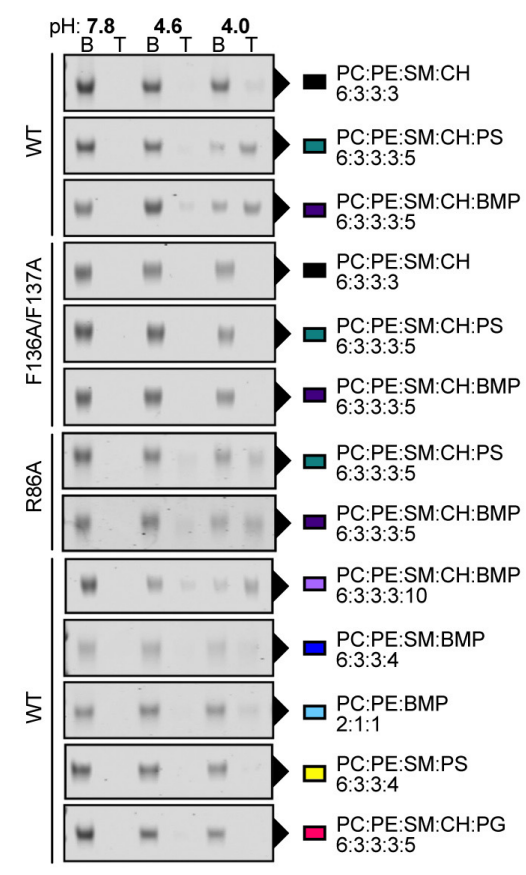

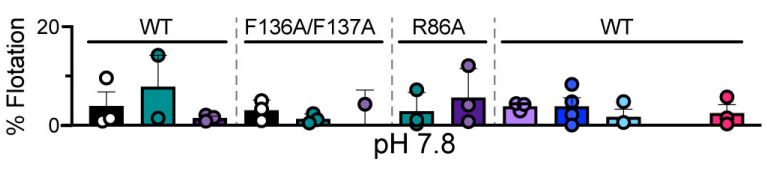
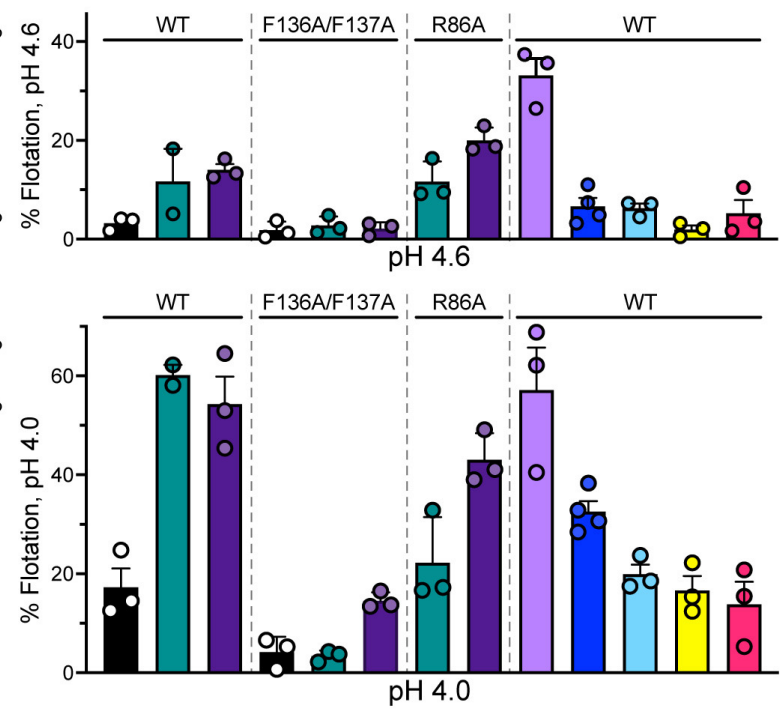

B

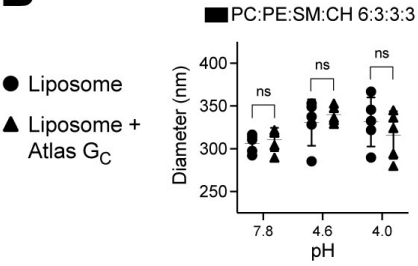

F136A/F137A Atlas $\mathrm{G}_{\mathrm{C}}$-liposome diameter

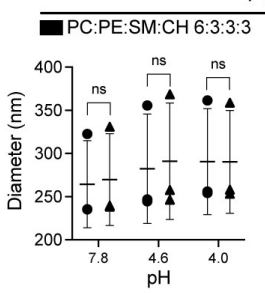

WT Atlas $\mathrm{G}_{\mathrm{C}}$-liposome diameter (DLS)

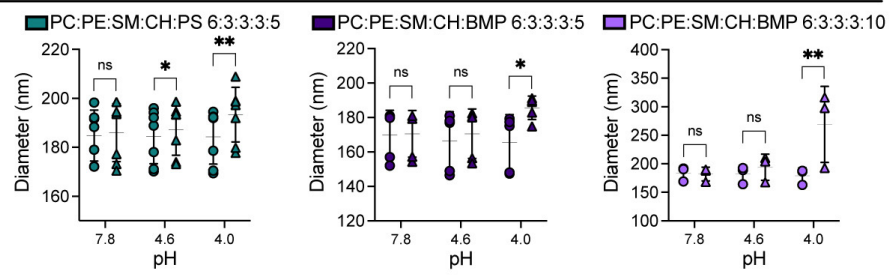

R86A Atlas $\mathrm{G}_{\mathrm{C}}$-liposome diameter
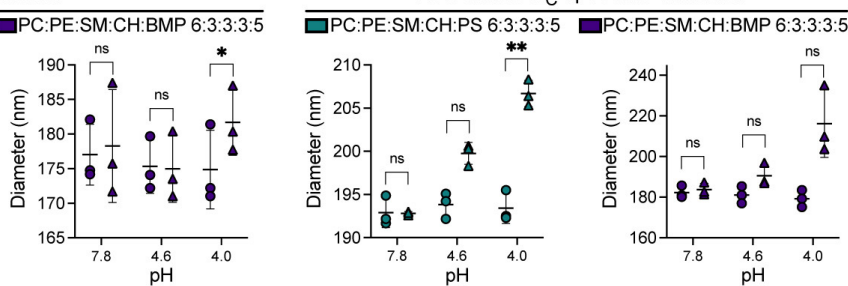

WT Atlas $\mathrm{G}_{\mathrm{C}}$-liposome diameter
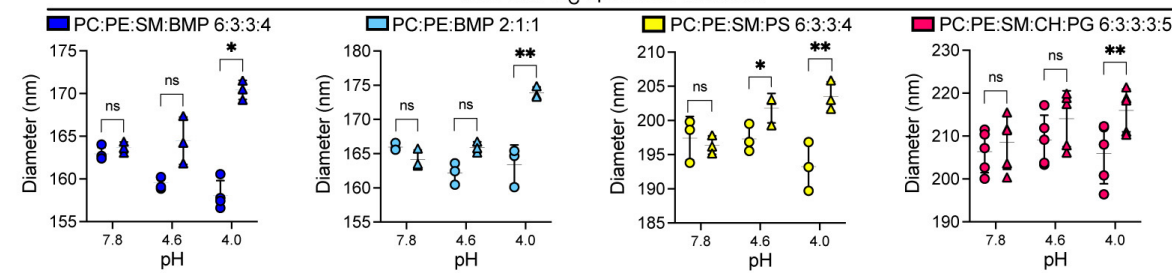

321 Fig. 4. Atlas $\mathbf{G}_{\mathbf{c}}$ binds liposomes in a lipid- and $\mathbf{p H}$-dependent manner. (A) Liposome co-

322 flotation lipid binding assay. A liposome- $\mathrm{G}_{\mathrm{c}}$ ectodomain mixture in $40 \%$ OptiPrep density

323 gradient medium was overlaid with a 30\% OptiPrep cushion and centrifuged at 100,000 g.

324 Flotation was defined as the amount of $\mathrm{G}_{\mathrm{c}}$ ectodomain co-floating with liposomes in the top-half

325 fraction ( $T$ ) divided by the total amount of $G_{c}$ ectodomain in the top- and bottom-half (B) 
326 fractions. $\mathrm{G}_{\mathrm{c}}$ ectodomain was quantified by Coomassie-stained SDS-PAGE. Error bars show

327 the standard deviation of three replicates except WT+PC:PE:SM:CH:PS (two replicates) and

328 WT+PC:PE:SM:BMP (four replicates). See fig. S8 for uncropped gels for all replicates. (B)

329 Binding of $\mathrm{G}_{\mathrm{c}}$ ectodomain (WT, F136A/F137A or R86A) to liposomes measured by dynamic

330 light scattering (DLS) as differences in liposome diameter in the presence and absence of Atlas

$331 \mathrm{G}_{\mathrm{c}}$ ectodomain. Error bars show the standard deviation of three to seven replicates.

332 Significance was determined by 2-way ANOVA of the mean change in liposome diameter, using

333 Sidak's multiple comparisons test with a 95\% confidence interval in GraphPad Prism 8 (see fig.

334 S7). ${ }^{* *}, 0.001<p<0.01 ;{ }^{*}, p<0.05 ;$ ns, not significant. See dataset $\mathbf{S} 1$ for source data.

335 Atlas $G_{c}$ membrane binding does not strictly require cholesterol and occurs via the

336 fusion loop

337 In addition to GPLs, phleboviruses and alphaviruses (but not flaviviruses) require cholesterol for

338 efficient membrane binding and subsequent fusion $(28,57,59)$. Alphaviruses additionally

339 require sphingolipids (such as SM) for efficient fusion $(60,61)$. We found that neither cholesterol

340 nor SM were required for Atlas $G_{c}$ to bind liposomes (Fig. 4, A and B). Removal of cholesterol

341 reduced the fraction of $\mathrm{G}_{c}$ bound by $50 \%$ in the liposome flotation assay (Fig. 4A) though

342 binding was still detected in the DLS assay (Fig. 4B). Hence, although cholesterol and SM are

343 not strictly required for binding, they enhance binding, possibly by increasing membrane fluidity.

344 Notably, the concentration of cholesterol in nematode cell membranes is approximately 20 times

345 lower than in vertebrates $(62,63)$. This is insufficient for cholesterol to regulate the structure or

346 fluidity of nematode membranes, in which cholesterol is thought to be instead a precursor for

347 low-abundance metabolites (62-64). Likewise, Drosophila can grow indefinitely with only trace

348 amounts of exogenous sterols (62), suggesting that arthropods, which are obligate vectors of

349 the vast majority of viruses containing class II fusion proteins, rely on lipids other than

350 cholesterol to regulate membrane fluidity. 
To determine whether Atlas $\mathrm{G}_{\mathrm{C}}$ binds membranes in a manner analogous to other viral

352 class II fusion proteins - via the fusion loop and GPL binding pocket - we purified Atlas $\mathrm{G}_{\mathrm{C}}$

353 ectodomain variants with mutations in the two phenylalanine residues in the fusion loop

354 (F136A/F137A), or in the arginine predicted to bind the GPL phosphate moiety (R86A). The

355 F136A/F137A mutant failed to bind liposomes containing PS and co-flotation with liposomes

356 containing BMP at pH 4 and $\mathrm{pH} 4.6$ was reduced to approximately one third of wild-type (Fig.

357 4C). The R86A mutation reduced binding to liposomes containing PS or BMP at $\mathrm{pH} 4$, to one

358 third and $75 \%$ of wild-type, respectively (Fig. 4D). For both variants, preparations contained

359 trimers as the major species but a small monomeric fraction was also present (fig. S2),

360 suggesting that the mutated lipid binding residues are required for efficient trimer assembly. We

361 conclude that Atlas $\mathrm{G}_{\mathrm{c}}$ binds to lipid membranes through insertion of hydrophobic fusion-loop

362 residues and coordination of lipid headgroups in the GPL binding pocket, as in other viral class

363 II fusion proteins.

\section{Evidence for monomeric and trimeric states of Atlas $\mathbf{G}_{c}$}

365 Class II fusion protein ectodomains can be monomeric, dimeric or form icosahedral shells in

366 their prefusion conformation, but the fusogenic conformational change is always accompanied

367 by reorganization into trimers $(26,57,65,66)$. Fusion proteins from classes I and III, including

368 retrovirus fusogens, remain trimeric throughout the fusion reaction, but no class II fusion

369 proteins are known to be trimeric in their prefusion conformation. Having established that Atlas

$370 \mathrm{G}_{\mathrm{c}}$ can insert into membranes as a trimer with a postfusion-like conformation, we set out to

371 determine whether it could undergo a conformational change as seen in the fusion reaction of

372 class II proteins from infectious viruses. The Atlas $\mathrm{G}_{\mathrm{c}}$ construct described above was expressed

373 as a trimer with no trace of monomers or dimers (Fig. 5A and fig. S2A). However, we found

374 that a construct with the stem region truncated, $\mathrm{G}_{\mathrm{C}}(\mathrm{DI}-\mathrm{III})$, spanning residues $2330-2751$, was

375 expressed as a mixture of monomers, trimers, and higher order oligomers (Fig. 5B and fig. 
376 S2B). In contrast to $\mathrm{G}_{\mathrm{C}}$ trimers, which were stable at different protein concentrations and $\mathrm{pH}$

377 values, $\mathrm{G}_{\mathrm{c}}(\mathrm{DI}-\mathrm{III})$ monomers were unstable over time. As noted above, monomeric fractions

378 were also present in preparations of the fusion loop mutant (F136A/F137A) and GPL binding

379 pocket mutant (R86A) (fig. S2, C and D). Whether these monomeric species are in a prefusion

380 conformation remains to be determined, but the presence of metastable monomers and stable

381 trimers recapitulates a key property of class II fusogens from infectious viruses.

$382 \mathrm{pH}-$ dependent stabilization of the Atlas $\mathbf{G}_{\mathrm{c}}$ trimer by protonated histidine residues

383 The increase in positive charge resulting from histidine protonation is an important part of the

$384 \mathrm{pH}$ sensing mechanism of viral class II fusion proteins. Protonation of conserved histidines at

385 the domain I-domain III interface of alphavirus, flavivirus and phlebovirus glycoproteins

386 promotes the fusogenic conformational change by destabilizing the prefusion conformation and

387 stabilizing the postfusion conformation (20, 28, 67-70). For example, histidines in domain III of

388 phlebovirus $\mathrm{G}_{c}$ proteins form interprotomer salt bridges with negatively charged side chains in

389 the postfusion trimer and mutation of one such histidine in RVFV, His1087, renders the virus

390 uninfectious (71). Similarly, in Atlas $\mathrm{G}_{\mathrm{C}}$ His258, His333 and His336 form interprotomer or

391 interdomain polar contacts (Fig. 5C). These histidine-dependent contacts would stabilize the

392 trimeric postfusion-like conformation of Atlas $\mathrm{G}_{\mathrm{C}}$ specifically in acidic endosomal compartments.

393 The parallels in how Atlas $G_{c}$ and class II fusogens from infectious viruses respond at the

394 ultrastructural level to environmental cues support the hypothesis that Atlas $G_{C}$ would have

395 membrane fusion activity in late endosomes, like phleboviruses and many retroviruses.

\section{Fifteen disulfide bonds stabilize Atlas $\mathbf{G}_{c}$ in its postfusion-like conformation}

397 With 30 cysteine residues forming 15 disulfide bonds, Atlas $\mathrm{G}_{c}$ contains twice the average

398 abundance of cysteines, more than has been found in any other class II protein. 20 of these

399 cysteines form disulfides that are structurally conserved in phleboviruses (including RVFV,

400 DABV and HRTV) but not in other class II proteins (fig. S5). An eleventh disulfide, in domain III, 
401 is conserved in Atlas virus, DABV and HRTV but not RVFV. However, Atlas $\mathrm{G}_{\mathrm{C}}$ contains four

402 additional disulfides not conserved in phleboviruses: one in the fusion loop, two in domain II in

403 the $\beta$-hairpin containing the $i j$ loop (one of the cysteines forming these disulfides is conserved in

404 phleboviruses but forms a disulfide with a cysteine in a different $\beta$-strand in domain II), and one

405 in domain III (Figs. 2C and 3A, and fig. S5). As discussed above, the Atlas-specific disulfide in

406 the fusion loop (Cys129-Cys138) extends the hydrophobic surface formed by conserved

407 residues in the fusion loop that are required for membrane insertion (Fig. 5D). We note that due

408 to the location of Cys129 and Cys138 close to the threefold symmetry axis of the trimer, the side

409 chains of the two residues can be rearranged by torsional rotation, to form intermolecular

410 disulfides across the trimer interface, thereby crosslinking all three protomers in the trimer.
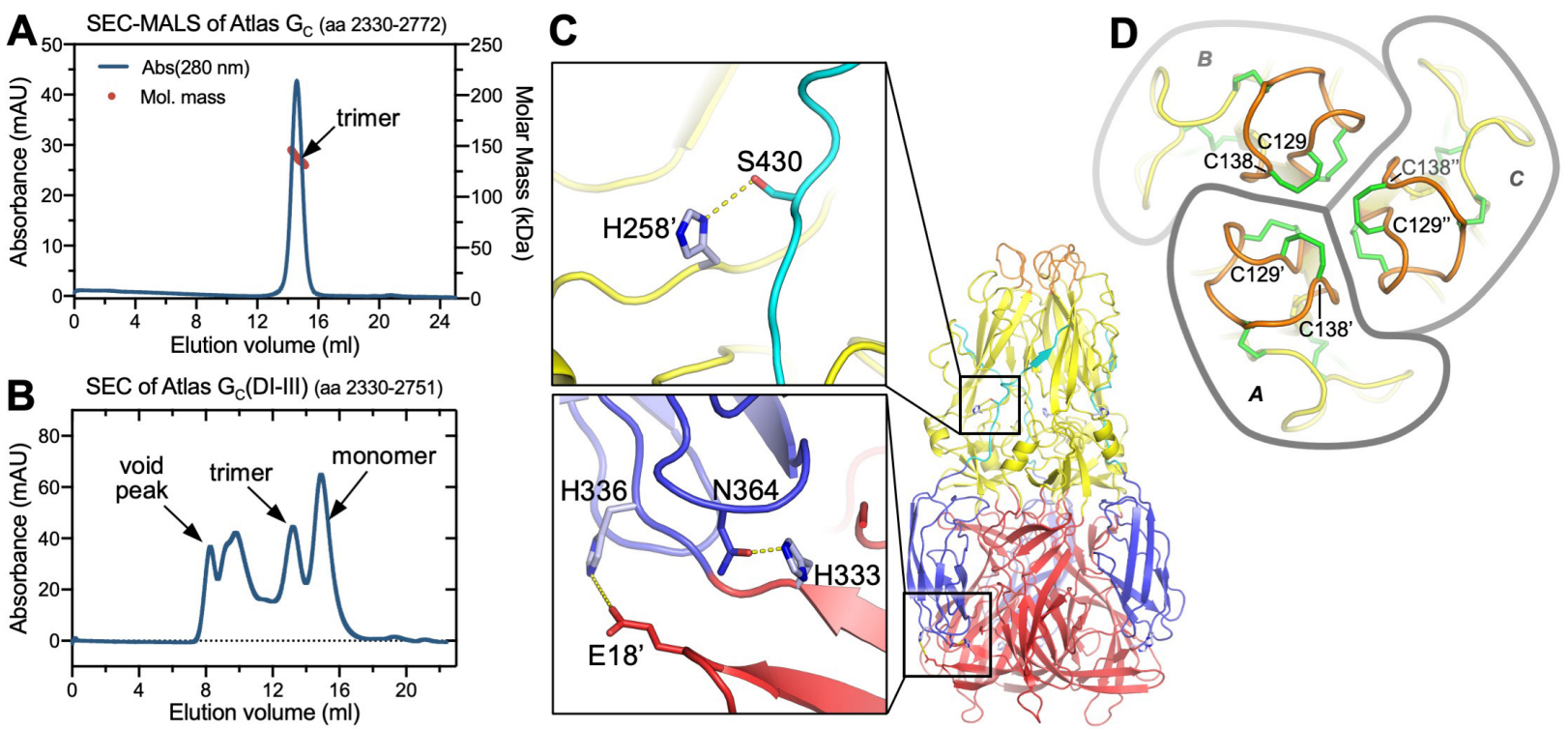

412 Fig. 5. Oligomeric states and disulfide bonding pattern of Atlas Gc. (A) SEC-MALS of Atlas

$413 \mathrm{G}_{c}$ ectodomain (residues 2330-2772). The protein formed trimers. (B) SEC of Atlas $\mathrm{G}_{c}(\mathrm{DI}-\mathrm{III})$

414 (residues 2330-2751) expressed in a mixture of oligomeric states, including monomers, trimers,

415 and larger aggregates (void peak). Gc(DI-III) monomers were unstable. (C) His258, His333 and

416 His336 form interprotomer or interdomain polar contacts predicted to stabilize the $\mathrm{G}_{\mathrm{c}}$ trimer

417 specifically at $\mathrm{pH}<6$, when the histidine side chains are charged. Residues from different 
418 protomers are denoted with a prime symbol. (D) View along the threefold axis of the $\mathrm{G}_{\mathrm{C}}$ trimer

419 with intramolecular disulfides between the cysteines most proximal to the axis, Cys129 and

$420 \quad$ Cys138.

\section{Atlas $\mathbf{G}_{c}$ retains membrane fusion activity}

422 Having identified the hallmarks of a fusion protein in Atlas $G_{c}$, we measured its membrane

423 fusion activity in a cell-cell fusion assay. $\mathrm{CHO}$ cells were transfected with plasmids encoding

424 Atlas $\mathrm{G}_{\mathrm{C}}$ or Atlas $\mathrm{G}_{\mathrm{N}}-\mathrm{G}_{\mathrm{C}}$. To promote plasma membrane localization and minimize $\mathrm{ER}$ retention,

425 we replaced the predicted transmembrane domain and cytosolic tail of $\mathrm{G}_{\mathrm{C}}$ with the C-terminal

426 transmembrane anchor and cytosolic tail from HLA-A2, known to localize to the plasma

427 membrane (72). Plasmids encoding vesicular stomatitis virus (VSV) G, or no protein, were used

428 as positive and negative controls, respectively. Since Atlas $\mathrm{G}_{\mathrm{c}}$ trimers require low $\mathrm{pH}$ and BMP

429 or PS to efficiently bind membranes, we treated transfected cells with exogenous BMP and then

430 transferred them to $\mathrm{pH} 4.5$ buffered medium to trigger fusion. Similar treatments have been

431 used previously to measure cell-cell fusion activity of flavi- and alphaviruses (53). Confocal light

432 microscopy with nuclear and plasma membrane stains showed that cells with three or more

433 nuclei were common in cells expressing Atlas $\mathrm{G}_{\mathrm{c}}$ following treatment with BMP and $\mathrm{pH} 4.5$,

434 though less abundant than in cells expressing VSV G, with or without treatment (Fig. 6, A and

435 B, and movie S1). Cells with more than three nuclei were rare in cells expressing Atlas $\mathrm{G}_{\mathrm{N}}-\mathrm{G}_{\mathrm{C}}$.

436 To quantify cell-cell fusion we counted nuclei and multinuclear cells in micrographs. We found

437 that in cells expressing Atlas $\mathrm{G}_{\mathrm{c}}$, the fraction of multinuclear cells, defined as cells containing

438 two or more nuclei, was $25 \pm 3 \%$ following treatment with BMP and pH 4.5, versus $10 \pm 0.2 \%$

439 without treatment (Fig. 6C; 95\% confidence intervals). Transfection of the $\mathrm{G}_{\mathrm{C}}$ fusion loop mutant

440 F136A/F137A resulted in essentially identical multinucleation fractions as with empty vector,

441 with or without treatment with BMP and $\mathrm{pH} 4.5$ (Fig. 6C), underpinning the importance of the

442 fusion loop in fusion activity. By comparison, in cells expressing VSV G, $73 \pm 8 \%$ of treated 
443 cells were multinuclear. $8 \pm 1 \%$ of cells transfected with empty vector were binuclear with or

444 without treatment, but none contained more than two nuclei (fig. S9). Incubation of transfected

445 cells with CDK4, which arrests the cell cycle at G1 phase, had no measurable effect on the

446 fraction of multinuclear cells (fig. S9D), indicating that cytokinesis effects do not significantly

447 contribute to this cell-cell fusion assay readout. We conclude that Atlas $\mathrm{G}_{\mathrm{c}}$ has approximately

448 one third of the membrane fusion activity of VSV G under the treatment conditions tested, which

449 is significant given that VSV G is considered highly fusogenic and widely used as a model

450 fusogen.

451 The Atlas $\mathrm{G}_{N}-\mathrm{G}_{\mathrm{C}}$ construct tested had little fusion activity (Fig. 6C). To investigate this,

452 we measured $\mathrm{G}_{\mathrm{C}}$ expression on the surface of cells transfected with the $\mathrm{G}_{\mathrm{N}}-\mathrm{G}_{\mathrm{C}}$ plasmid. Cell-

453 surface proteins were biotinylated, affinity-purified and analyzed by immunoblot and liquid

454 chromatography with tandem mass spectrometry (LC-MS/MS). When expressed as part of a

$455 \mathrm{G}_{\mathrm{N}}-\mathrm{G}_{\mathrm{C}}$ construct, only trace levels of cell-surface $\mathrm{G}_{\mathrm{C}}$ were detected by Western blot (Fig. 6D).

456 Moreover, no $\mathrm{G}_{\mathrm{N}}$ peptides were observed by LC-MS/MS, and the LC-MS/MS signal for $\mathrm{G}_{\mathrm{C}}$ was

457 significantly weaker than in cells transfected with $\mathrm{G}_{\mathrm{c}}$ alone (Fig. 6E), consistent with the lack of

458 fusion activity in cells transfected with $\mathrm{G}_{N}-\mathrm{G}_{\mathrm{C}}$. We conclude that the $\mathrm{G}_{\mathrm{N}}$ was degraded or failed

459 to be secreted in $\mathrm{CHO}$ cells, which in turn prevented efficient secretion of $\mathrm{G}_{\mathrm{c}}$. 

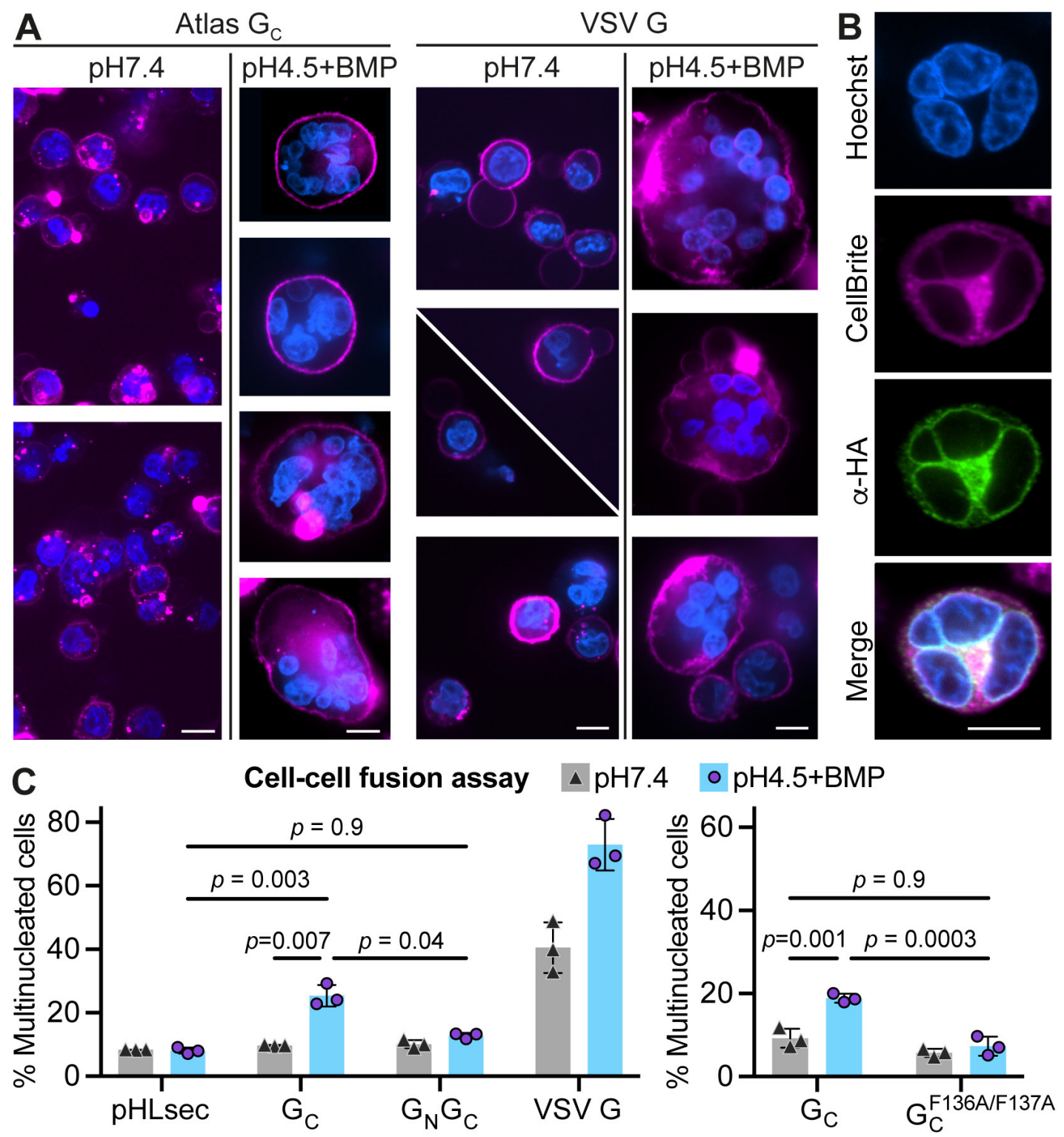

D

$E$

\begin{tabular}{l}
$\begin{array}{c}\text { Cell-surface } \\
\text { biotinylation } \\
\text { Western blot }\end{array}$ \\
\cline { 2 - 9 } \\
\cline { 2 - 9 }
\end{tabular}

Fig. 6. Cell-cell fusion assay. (A) Confocal micrographs of $\mathrm{CHO}$ cells expressing Atlas $\mathrm{G}_{\mathrm{C}}$

462 fused to the transmembrane anchor from HLA-A2, or VSV G. Cells were treated with $\mathrm{pH} 4.5$ 
463 buffer containing BMP, or pH 7.4 buffer without lipid. Blue, Hoechst 33342 nuclear stain;

464 Magenta, CellBrite Red plasma membrane dye. Micrographs were selected to show fusion

465 events - see fig. $\mathbf{S 9}$ for representative raw micrographs. Scale bars, $10 \mu \mathrm{m}$. (B) Confocal

466 micrograph of a multinucleated $\mathrm{CHO}$ cell expressing Atlas $\mathrm{G}_{\mathrm{c}}$ following treatment with $\mathrm{pH} 4.5$

467 and BMP. Blue, Hoechst 33342; magenta, CellBrite Red; green, a-HA antibody for Atlas $\mathrm{G}_{\mathrm{c}}$

468 detection. Scale bar, $10 \mu \mathrm{m}$. A composite Z-stack of this cell is shown in movie S1. (C) The

469 fraction of multinuclear cells transfected with plasmids encoding Atlas $\mathrm{G}_{\mathrm{C}}$, Atlas $\mathrm{G}_{\mathrm{N}}-\mathrm{G}_{\mathrm{C}}$, VSV G

470 or pHLsec empty vector was calculated by counting mono- and multi-nucleated cells using the

471 Hoechst and CellBrite stains. Error bars represent SD between measurements from three

472 distinct experiments, with 135-407 nuclei counted per replicate. $P$-values were calculated by $2-$

473 way ANOVA (Tukey's multiple comparisons test, GraphPad Prism 9). (D and E) Gc cell-surface

474 expression analysis. Cells were biotinylated, cell-surface proteins were affinity-purified with

475 NeutrAvidin agarose, and the cell-surface $\mathrm{G}_{\mathrm{c}}$ fraction was quantified by Western blot, (D), and

476 LC-MS/MS mass spectrometry, (E). See dataset S2 for source data.

477 Transcriptional activity of Atlas virus based on RNA-Seq data

478 Transcription of endogenous viruses is tightly regulated to prevent harmful gene expression and

479 genome damage from transposition events. However, some EVEs escape repression and are

480 highly transcribed, including a subset of belpaoviruses from the parasitic trematode

481 Schistosoma mansoni (blood fluke) (73). We analyzed transcript levels of Atlas and other

482 complete belpaoviruses in previously published RNA-Seq data for A. ceylanicum (74).

483 Transcript abundance of the belpaovirus EVEs encoding complete polyproteins varied, ranging

484 from undetectable to above the median value for all annotated genes (Fig. 7 and fig. S10). For

485 Atlas virus, transcript levels varied across developmental stages: expression was above the

486 median for annotated genes at the larval L3 stage, below the first quartile at stage L4, and near

487 the median in adult hookworms (Fig. 7A). Notably, Atlas virus transcript expression increased 

differential expression calculations).
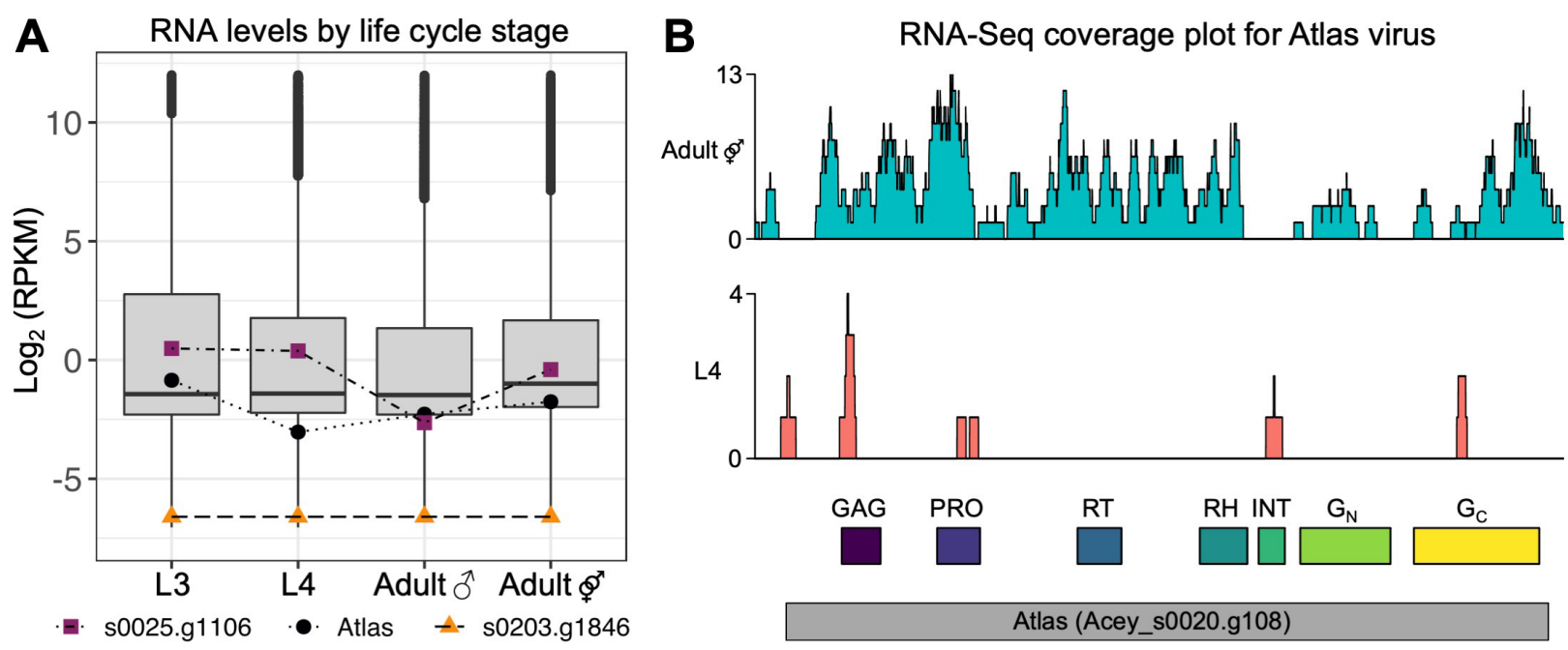

491 Fig. 7. RNA-Seq signal from Atlas virus and other A. ceylanicum genes. (A) Box plots of

492 RNA-Seq Reads Per Kilobase of transcript per Million mapped reads (RPKM) for all annotated

493 A. ceylanicum genes, at different developmental stages (L3 and L4 larva, adult male and adult

494 mixed sex). RNA-Seq data are from Bernot et al. (74). Bars in each box represent the median.

495 Box boundaries represent the first and third quartiles. RPKM values are shown for Atlas virus

496 and the complete belpaovirus EVEs with the highest and lowest RNA-Seq signals, s0025.g1106

497 and s0203.g1846, respectively. (B) Distribution of RNA-Seq reads over the Atlas virus coding

498 sequence for the adult (mixed sex) and L4 developmental stages. Read coverage was greater

499 in adults. See fig. $\mathbf{S 1 0}$ for a snapshot of this region from Integrative Genomics Viewer with

500 unmerged BAM files.

\section{Discussion}

502 Here, we identify Atlas virus as an endogenous belpaovirus containing intact gag, pol and env

503 genes with novel features in the human hookworm A. ceylanicum. The cryo-EM structure of the

504 Atlas Env reveals a class II viral fusion protein fold remarkably similar to that of the $\mathrm{G}_{\mathrm{c}}$

505 glycoprotein from Rift Valley Fever virus. Atlas $\mathrm{G}_{\mathrm{C}}$ has the hallmarks of an active class II 
506 membrane fusion protein: a stable trimeric assembly, a putative fusion loop, membrane

507 insertion triggered by low $\mathrm{pH}$ with specificity for late endosomal lipid composition, and

508 membrane fusion activity. Moreover, Atlas virus mRNA is expressed at specific stages of

509 hookworm development.

510 Our work supports the model first proposed based on phylogenetic studies that the

511 nematode belpaoviruses acquired their Env by horizontal gene transfer from a virus from the

512 Phenuiviridae family or a phlebovirus-like ancestor (14). It remains unclear whether RNA

513 encoding the phlebovirus-like glycoprotein integrated into the belpaovirus ancestor as mRNA in

514 a splicing event, or by first becoming a substrate for the reverse transcriptase with subsequent

515 genomic integration as dsDNA. The envelope proteins from retroviruses, including ERVs, that

516 have been biochemically characterized were all found to be class I fusion proteins with an $\alpha-$

517 helical coiled-coil as the core fold. Viral class II fusion proteins have so far been found only in

518 non-integrating RNA viruses. Our discovery of an endogenous belpaovirus with a potentially

519 functional, phlebovirus-like class II fusion protein that is structurally unrelated to retrovirus Envs

520 reveals an unexpected degree of structural and genetic plasticity in reverse-transcribing RNA

521 viruses. More generally, the presence of the class II fusion protein fold in EVEs and across

522 many phyla suggests this fold is derived from a common ancestor that could have been viral or

523 cellular in origin $(33-36,75)$.

525 While rare, horizontal gene transfer of atypical fusogens into retroelements is not unique

526 to the belpaoviruses. The Tas element from the nematode Ascaris lumbricoides, a common

527 parasitic worm in humans, has an env gene with weak genetic similarity to herpesvirus gB

528 proteins (14), which have a class III fusion protein fold (also found in Rhabdoviridae and

529 Baculoviridae). Taken together, these findings lead us to hypothesize that acquisition of a fusion

530 protein from an infectious virus represents a general paradigm for how retrotransposons can 
531 become retroviruses, and indeed how ancestral reverse-transcribing viruses may have

532 originated.

533 The env gene is often the first element to be lost in EVEs, as it is not required for

534 intracellular proliferation, so it is notable that the $\mathrm{G}_{N}-\mathrm{G}_{\mathrm{C}}$ env module is intact in Atlas virus. With

535 its identical LTRs, and no stop codons or frameshift mutations, Atlas virus shows all the signs of

536 being intact and recently active. This supports the notion that the envelope may be functional

537 (12). The preserved biological activities of Atlas $G_{c}$ suggest these activities could have cellular

538 functions in health and disease, as reported for a small but increasing number of ERV env and

539 gag gene products $(1,3,5,76)$. Further studies are required to determine the full extent to

540 which protein expression from transposable elements - and its dysregulation - contribute to

541 basic cellular functions, embryonic development, and disease outcomes. This work provides a

542 blueprint for such efforts.

\section{Materials and Methods}

544 Genomic analyses of $\boldsymbol{A}$. ceylanicum Atlas virus. A PSI-BLAST search (40) for protein

545 sequences similar to biochemically characterized phlebovirus fusion proteins identified the gene

546 Acey_s0020.g108 (UniProt A0A016UZK2; Genbank JARK01001356.1, genomic translation

547 EYC20859.1) in the human hookworm Ancylostoma ceylanicum as the most similar sequence

548 outside infectious virus taxa, with an Expected value (E-value) of $10^{-20}$ against the Rift Valley

549 Fever virus (RVFV) $G_{c}$ sequence (UniProt P03518). A second iteration performed using

550 position-specific scoring matrix based on an alignment of sequences identified in the first

551 iteration gave an E-value of $10^{-144}$. The Acey_s0020.g108 gene is referred to here as Atlas

552 virus.

553 Phylogenetic analysis of the phlebovirus $\mathrm{G}_{c}$-like protein from Atlas virus and other $\mathrm{G}_{c}$ or

$554 \mathrm{G}_{\mathrm{C}}$-like proteins with similar protein sequences was performed as follows. A reference multiple

555 sequence alignment was initially generated by aligning the Atlas $\mathrm{G}_{c}$ protein sequence with the 
556 sequences of reference protein $R V F V G_{c}$ and other viral $G_{c}$ proteins identified as having

557 structural similarity in structure comparisons with DALI (50), namely the $\mathrm{G}_{c}$ proteins from Dabie

558 bandavirus (DABV, formerly SFTSV phlebovirus, GenBank:AGM33042.1) and Heartland virus

559 (HRTV, GenBank:AFP33394.1). For each $\mathrm{G}_{c}$ protein, the sequence range present in the

560 corresponding Protein Data Bank (PDB) entry (7A4A, 6EGU, 5G47, or 5YOW, respectively) was

561 defined as the $G_{C}$ ectodomain sequence and used to generate the reference alignment with

562 MAFFT in SnapGene v5.1.7. The sequences of the following EVEs with detectable Env protein

563 sequence similarity were then added to the alignment: Necator americanus NECAM_13468

564 (GenBank:XM_013440329.1), C. elegans Cer13 virus (GenBank: Z81510.2; WormBase

565 WBTransposon00000728, gene Y75D11A.5), and seven A. ceylanicum EVEs encoding

566 complete Gag-Pol-Env polyproteins with predicted phlebovirus-like Envs (Fig. 1B; GenBank

567 EYC27361.1, EYC19962.1, EYC20099.1, EYC18998.1, EYC07469.1, EYB91703.1,

568 EYB80846.1). The tree was calculated with IQ-TREE v1.6.12 and drawn with iTOL v6 (44).

569 The reverse transcriptase (RT) phylogenetic tree was calculated based on the protein

570 sequences of Atlas virus, Cer13 virus, the seven $A$. ceylanicum EVEs listed above, and seven

571 other belpaoviruses (GenBank AF060859.1, Z29712.1, L09635.1, U23420.1, AY180917.1,

572 D83207.1, AF537216.1). RT domains (Pfam:PF00078, RVT_1) were identified with PfamScan

573 in five of the sequences (GenBank Z81510.2, EYC27361.1, EYC19962.1, EYC07469.1,

574 EYB80846.1). The protein sequences of these five RTs were extracted and aligned with MAFFT

575 and Clustal Omega v1.2.4. The tree was generated with IQ-TREE v1.6.12 and iTOL v6 (44).

576 The Atlas virus LTRs were identified with REPuter (77). Sequence identity matrices (fig.

577 S1) were calculated with SDT (78).

578 Protein expression and purification. Synthetic genes encoding soluble ectodomain fragments

579 of the Env of endogenous retrovirus Y032_0020g108 from A. ceylanicum were subcloned into 
580 the pMT/BiP/V5-His vector (ThermoFisher) in frame with the BiP signal sequence and the C-

581 terminal V5 and six-histidine tags. The constructs referred to here as Atlas $\mathrm{G}_{c}$ and Atlas $\mathrm{G}_{\mathrm{c}}(\mathrm{DI}-$

582 III) span amino acids 2330-2772 and 2330-2751 from UniProt A0A016UZK2, respectively. Atlas

$583 \mathrm{G}_{\mathrm{c}}$ mutants were generated by Dpn I-based site-directed mutagenesis. D.mel-2 insect cells

584 (ThermoFisher) were co-transfected with the expression construct and blasticidin resistance

585 marker pCoBlast (ThermoFisher) at a 20:1 molar ration and cultured for 6 weeks in $0.5 \mu \mathrm{ml}^{-1}$

586 blasticidin to obtain a population of expressor cells. Expression was induced in a shaking cell

587 suspension at $27^{\circ} \mathrm{C}$ with $0.5 \mathrm{mM} \mathrm{CuSO}_{4}$ at a cell density of $5 \times 10^{6}$ cells ml${ }^{-1}$. The cell culture

588 medium was harvested 4 to 5 days after induction, centrifuged to remove cells $(2,000 \mathrm{~g})$ and cell

589 debris $(17,000 \mathrm{~g})$, filtered with a $0.2 \mu \mathrm{m}$ filter, concentrated by tangential-flow filtration, and

590 buffer exchanged into $20 \mathrm{mM}$ Tris $\mathrm{pH}$ 7.8, $0.3 \mathrm{M} \mathrm{NaCl}, 5 \%$ glycerol, $20 \mathrm{mM}$ imidazole, $0.5 \mathrm{mM}$

591 TCEP (tris(2-carboxyethyl)phosphine). Atlas $\mathrm{G}_{\mathrm{c}}$ was purified by nickel-affinity chromatography

592 with a HisTrap Excel column (Cytiva), followed by anion-exchange chromatography with a

593 MonoQ or Resource Q column (Cytiva) using 20 mM Tris pH 8.0, 50 mM NaCl, 5\% glycerol, 0.5

$594 \mathrm{mM}$ TCEP as the binding buffer and binding buffer plus $1 \mathrm{M} \mathrm{NaCl}$ as the elution buffer. Peak

595 fractions were concentrated and further purified by size-exclusion chromatography (SEC) with a

596 Superdex 200 Increase (10/300) column (Cytiva) in $20 \mathrm{mM}$ Tris pH 7.8-8.0, $0.15 \mathrm{M} \mathrm{NaCl}, 5 \%$

597 glycerol and $0.5 \mathrm{mM}$ TCEP. The C-terminal V5 and histidine tags were optionally cleaved by

598 incubation with carboxypeptidase $\mathrm{A}(\mathrm{CPA})$ for $3 \mathrm{~h}$ at $4^{\circ} \mathrm{C}\left(1: 500 \mathrm{CPA}: \mathrm{G}_{\mathrm{C}}\right.$ molar ratio).

599 Liposome binding assay. 1-palmitoyl-2-oleoyl-sn-glycero-3-phosphocholine (PC), 1-palmitoyl-

600 2-oleoyl-snglycero-3-phosphoethanolamine (PE), egg sphingomyelin (SM), 1-palmitoyl-2-oleoyl-

601 sn-glycero-3-phospho-L-serine (PS), 1-palmitoyl-2-oleoyl-sn-glycero-3-phospho-(1'-rac-glycerol)

602 (PG), (S,R) Bis(Monoacylglycero)Phosphate (BMP) (Avanti Polar Lipids), and 1-cholesterol

603 (Sigma-Aldrich) were dissolved in chloroform. $25 \mathrm{mM}$ lipid solutions were mixed at various molar

604 ratios and dried under nitrogen gas for over $4 \mathrm{~h}$. The lipid film was resuspended in Liposome 
605 Buffer (20 mM Tris pH 7.8, 0.15 M NaCl, 5\% glycerol, 0.5 mM TCEP, 2 mM MgCl $2,2 \mathrm{mM} \mathrm{CaCl}_{2}$,

$6062 \mathrm{mM} \mathrm{KCl}$ ) and subjected to five cycles of freeze-thawing in liquid nitrogen, followed by 25

607 cycles of extrusion through two $0.2 \mu \mathrm{m}$ polycarbonate filter membranes (Whatman). Purified

608 Atlas $\mathrm{G}_{\mathrm{c}}$ ectodomain was added in a 1:771 protein:lipid molar ratio and incubated at $37^{\circ} \mathrm{C}$ for 5

609 min. The $\mathrm{pH}$ was reduced by adding a $2 \mathrm{M}$ stock solution of sodium acetate $\mathrm{pH} 4.6$ or 4.0 to a

610 final concentration of $0.2 \mathrm{M}$. Following a $2 \mathrm{~h}$ incubation at $37^{\circ} \mathrm{C}$ the $\mathrm{pH}$ of the suspension was

611 neutralized with $1 \mathrm{M}$ Tris $\mathrm{pH}$ 8. OptiPrep density gradient medium (Sigma-Aldrich) was added to

612 a concentration of $40 \%$, maintaining $0.15 \mathrm{M} \mathrm{NaCl}$ throughout. Approximately $0.5 \mathrm{ml}$ of the

613 liposome suspension was placed in a centrifuge tube, overlayed with a $2.5 \mathrm{ml}$ cushion of $30 \%$

614 OptiPrep solution and centrifuged at $100,000 \mathrm{~g}$ for $1 \mathrm{~h}$ at $4^{\circ} \mathrm{C}$ in a TLA100.3 rotor (Beckman

615 Coulter). Top ( $\mathrm{T}$ ) and bottom (B) fractions (approximately $1.5 \mathrm{ml}$ each) were collected from the

616 top meniscus with a micropipette. Atlas $\mathrm{G}_{\mathrm{c}}$ was quantified by densitometry of the absorbance at

$617700 \mathrm{~nm}$ of bands in Coomassie-stained SDS-PAGE gels with an Odyssey scanner (LI-COR).

618 Flotation was defined as the amount of Atlas $\mathrm{G}_{c}$ ectodomain in the top fraction divided by the

619 total amount of Atlas $\mathrm{G}_{\mathrm{c}}$ ectodomain in both fractions.

620 For measurement of liposome diameter by dynamic light scattering (DLS), the liposome

621 suspensions were diluted ten-fold in Liposome Buffer prior to the addition of $40 \%$ OptiPrep

622 solution. Following centrifugation liposome diameters were measured in 384-well clear-

623 bottomed optical imaging plates (Corning) with a DynaPro Plate Reader III (Wyatt

624 Technologies). The mean diameter was calculated as the average of three independent

625 measurements, each consisting of fifteen two-second acquisitions. Protein-free acidified

626 liposome controls were treated and measured in parallel, with Liposome Buffer instead of Atlas

$627 \mathrm{G}_{c}$ solution.

628 Size-exclusion chromatography and multi-angle scattering (SEC-MALS) analysis. $100 \mu \mathrm{l}$

629 samples containing $1.6-2.5 \mathrm{mg} \mathrm{ml}^{-1}$ Atlas $\mathrm{G}_{c}$ ectodomain were analysed by size-exclusion 
630 chromatography (SEC) at $293 \mathrm{~K}$ on a Superdex 200 (10/300) column (Cytiva) in $20 \mathrm{mM}$ Tris pH

$6317.8,0.15 \mathrm{M} \mathrm{NaCl}, 5 \%$ glycerol and $0.5 \mathrm{mM}$ TCEP with a flow rate of $0.5 \mathrm{ml} \mathrm{min}^{-1}$. The SEC

632 system was coupled to both multi-angle light scattering (MALS) and quasi-elastic light scattering

633 (QELS) modules (DAWN-8+, Wyatt Technology). The protein was also detected as it eluted

634 from the column with a differential refractometer (Optilab T-rEX, Wyatt Technology) and a UV

635 detector at $280 \mathrm{~nm}$ (Agilent 1260 UV, Agilent Technology). Molar masses of peaks in the elution

636 profile were calculated from the light scattering and protein concentration, quantified using the

637 differential refractive index of the peak assuming a dn/dc of 0.1860 , with ASTRA6 (Wyatt

638 Technology).

639 Cryo-EM sample preparation and data collection. Purified Atlas $\mathrm{G}_{c}$ ectodomain trimer (3 $\mu \mathrm{l}$

640 at a concentration of $0.025 \mathrm{mg} \mathrm{ml}^{-1}$ ) in $20 \mathrm{mM}$ Tris $\mathrm{pH} 7.8,0.15 \mathrm{M} \mathrm{NaCl}, 5 \%$ glycerol and 0.5

641 mM TCEP, was applied onto glow-discharged R1.2/1.3 400 mesh copper grids (Quantifoil Micro

642 Tools, Germany). The grids were blotted for $4 \mathrm{~s}$ and plunge-frozen in liquid ethane with a

643 Vitrobot Mark IV (ThermoFisher) at $4^{\circ} \mathrm{C}$ and $100 \%$ humidity. Preliminary sample screening and

644 initial datasets were acquired on a FEI Tecnai F20 microscope operated at $200 \mathrm{kV}$ equipped

645 with Falcon II direct electron detector (ThermoFisher) at $-4 \mu \mathrm{m}$ defocus. High-resolution cryo-EM

646 dataset collection was performed on a Titan Krios microscope (ThermoFisher) operated at

$647300 \mathrm{kV}$ equipped with a $20 \mathrm{eV}$ slit-width GIF quantum energy-filtered Gatan K2-Summit direct

648 electron detector in counting mode. A total of 3,027 movies were recorded at a calibrated

649 magnification of $130,000 x$ leading to a magnified pixel size of $1.047 \AA$ on the specimen. Each

650 movie comprised 36 frames with an exposure rate of $1.28 \mathrm{e}^{-} \AA^{-2}$ per frame, with a total exposure

651 time of $8 \mathrm{~s}$ and an accumulated exposure of $46.18 \mathrm{e}^{-} \AA^{-2}$. Data acquisition was performed with

652 EPU Automated Data Acquisition Software for Single Particle Analysis (ThermoFisher) with

653 three shots per hole at $-1.3 \mu \mathrm{m}$ to $-3.5 \mu \mathrm{m}$ defocus. 
654 Image processing. Micrographs from initial datasets allowed us to obtain a consistent model at

$655 \sim 19 \AA$ A resolution from 3,790 particles selected after 2D and 3D classification, and consequent

656 auto-refinement. All movies from high-resolution datasets were motion-corrected and dose-

657 weighted with MOTIONCOR2 (79). Aligned, non-dose-weighted micrographs were then used to

658 estimate the contrast transfer function (CTF) with GCTF (80). All subsequent image processing

659 steps were performed using RELION $3.0(81,82)$. 2D references from initial datasets were used

660 to auto-pick the micrographs. One round of reference-free $2 \mathrm{D}$ classification was performed to

661 produce templates for better reference-dependent auto-picking, resulting in a total of 987,570

662 particles. After a first round of 2D classification, 595,011 particles were selected to perform a

663 second 2D classification, resulting in a final number of 320,041 selected particles. Then, a 3D

664 classification imposing C3 symmetry was performed using the model from the initial datasets

665 filtered at $40 \AA$ resolution as initial model. The best class, containing 197,145 particles, was

666 selected, and subjected to 3D auto-refinement imposing C3 symmetry, yielding a map with an

667 overall resolution at $4.11 \AA$ based on the gold-standard $(F S C=0.143)$ criterion. After

668 refinement, the CTF refinement (per-particle defocus fitting and beam tilt estimation) and

669 Bayesian polishing (83) routines implemented in RELION 3.0 were performed, yielding a final

670 map with an overall resolution at 3.76 A. Local resolution was estimated with RELION.

671 Model building and refinement. The most similar sequence to Atlas $\mathrm{G}_{c}$ with a structure

672 available was glycoprotein $\mathrm{G}_{\mathrm{C}}$ from Rift Valley Fever virus (RVFV). The crystal structure of

673 RVFV $G_{c}$ in the postfusion conformation (PDB:6EGU (28)) was used as template to build a

674 homology model with the sequence of Atlas $\mathrm{G}_{c}$ using the Swiss-Model server

675 (https://swissmodel.expasy.org). The output model was docked as a rigid body into the density

676 with UCSF Chimera (84). Initial docking was performed manually and was followed by real

677 space fitting with the Fit in Map routine. A preliminary step of real space refinement was

678 performed on the three-subunit model, with Phenix 1.13 (85), with global minimization, atomic 
679 displacement parameter (ADP), simulated annealing and morphing options selected. The model

680 was then rebuilt in Coot (86) to optimize the fit to the density. Due to low resolution information

681 in the fusion loop region, the density was converted to .mtz file using CCP-EM software

682 package tools and blurring of the density allowed us to localize bulky residues and disulphide

683 bonds, and thus use them as a guide to build the entire fusion loop. A final step of real space

684 refinement was performed with Phenix 1.15, with global minimization and ADP options selected.

685 The following restraints were used in the real space refinement steps: secondary structure

686 restraints, non-crystallographic symmetry (NCS) restraints between the protein subunits, side

687 chain rotamer restraints, and Ramachandran restraints. Key refinement statistics are listed in

688 table S1.

689 Model validation and analysis. The FSC curve between the final model and full map after

690 post-processing in RELION, Model vs Map, is shown in fig. S3. Cross-validation against

691 overfitting was performed as described (87). The atoms in the final atomic model were displaced

692 by $0.5 \AA$ in random directions with Phenix. The shifted coordinates were then refined against

693 one of the half-maps generated in RELION, the "work set". This test refinement was performed

694 in Phenix using the same procedure as for the refinement of the final model (see above). The

695 other half-map, the "test set" was not used in refinement for cross-validation. FSC curves of the

696 refined shifted model against the work set, FSCwork, and against the test set, FSCtest, are

697 shown in fig. S3. The FSCwork and FSCtest curves are not significantly different, consistent

698 with the absence of overfitting in our final models.

699 The quality of the atomic models, including basic protein geometry, Ramachandran

700 plots, clash analysis, was assessed and validated with Coot, MolProbity (88) as implemented in

701 Phenix 1.15, and with the Worldwide PDB (wwPDB) OneDep System (https://deposit-

702 pdbe.wwpdb.org/deposition). 
703 Cell-cell fusion assay. Chinese hamster ovary $(\mathrm{CHO})$ Lec3.2.8.1 cells were transfected with

704 pHL-Sec plasmids encoding ectodomain fragments of Atlas $\mathrm{G}_{\mathrm{N}}-\mathrm{G}_{\mathrm{C}}$ (polyprotein residues 1909-

7052795 ) or Atlas $\mathrm{G}_{\mathrm{C}}$ (residues 2330-2795) fused to the C-terminal transmembrane domain from

706 HLA-A2 (residues 288-345) and cloned in frame with the vector's secretion signal and a C-

707 terminal HA tag. Empty pHL-Sec plasmid, and pcDNA encoding vesicular stomatitis virus (VSV)

708 G, were used as negative and positive controls, respectively. 16-20 h post-transfection, cells

709 were transferred to PBS supplemented with $2.5 \mathrm{mM}$ BMP $(18: 1(S, S)$

710 Bis(Monoacylglycero)Phosphate (BMP); Avanti Polar Lipids). To obtain a homogeneous BMP

711 suspension, the mixture was freeze-thawed five times using liquid nitrogen and a water bath,

712 followed by a 3 min incubation in a sonicating water bath. Cells were incubated in the BMP

713 suspension (or PBS for the untreated control) at $37^{\circ} \mathrm{C}$ for $5 \mathrm{~min}$, shown previously to be

714 sufficient for anionic lipid incorporation into the plasma membrane (53). Cell were transferred to

$715 \mathrm{pH} 4.5$ complete medium (DMEM adjusted to $\mathrm{pH} 4.5$ with $\mathrm{HCl}$ supplemented with $10 \%$ foetal

716 bovine serum), or $\mathrm{pH} 7.4$ complete medium for the untreated control, and centrifuged at $2,500 \mathrm{~g}$

717 at $37^{\circ} \mathrm{C}$ for $2 \mathrm{~min}$. Cells were immediately resuspended in complete media and plated out.

718 Following reattachment (4-6 h after treatment), cells were stained with Hoechst 33342 (Bio-Rad)

719 and CellBrite Red cytoplasmic membrane dye (Biotium, cat. no. 30023) and imaged on a Nikon

720 iSIM Swept Field inverted confocal microscope with a 60x/1.2NA water objective. Cluster

721 analysis of the Hoechst channel with Fiji (89) was used to count single nuclei and identify

722 polynuclear clusters. Nuclei within polynuclear clusters were counted by visual inspection. The

723 plasma membrane stain was used to confirm polynuclear clusters and count the number of

724 multinuclear cells, defined as cells with two or more nuclei, by visual inspection. The fraction of

725 multinucleated cells $(F)$ was calculated using the formula:

726

$F=1-($ n.mononucleated cells $) /($ total $n . n u c l e i)$ 
For testing the effect of inhibiting the cell cycle on the fraction of multinucleated cells,

728 cells were transfected with plasmid pEGFP-N1, and treated on the following day with $10 \mu \mathrm{M}$

729 CDK4 (Cayman Chemical, Cat. No. 17648), or PBS in the negative control, for $6 \mathrm{~h}$ at $\mathrm{pH} 7.4$.

730 Cell surface biotinylation and mass spectrometry. Proteins on the cell surface were

731 biotinylated and isolated with the Pierce ${ }^{\mathrm{TM}}$ Cell Surface Protein Biotinylation and Isolation Kit

732 (ThermoFisher, A44390). HEK293T cells were transfected with $3 \mu \mathrm{g}$ pHLsec-G $\mathrm{NG}_{\mathrm{c}}$, or pHLsec-

733 G. One day later, cells were washed with PBS and incubated with $0.25 \mathrm{~g} / \mathrm{l}$ Sulfo-NHS-SS-Biotin

734 on ice for $10 \mathrm{~min}$. Then cells were washed with PBS and suspended in $0.5 \mathrm{ml}$ of ThermoFisher

735 Lysis Buffer supplemented with cOmplete EDTA-free Protease Inhibitor Cocktail (Merck). Cells

736 were lysed on ice for 30 min (with 5s vortexing before and after lysis) and centrifuged at 15,000

$737 \times \mathrm{g}$ for $5 \mathrm{~min}$ at $4^{\circ} \mathrm{C}$. The lysate supernatant was incubated with $0.25 \mathrm{ml}$ NeutrAvidin Agarose

738 resin for $30 \mathrm{~min}$ at $20^{\circ} \mathrm{C}$ on an end-over-end rotator. The resin was washed with Wash Buffer,

739 and bound proteins eluted Elution Buffer from the kit

740 Samples were prepared for mass spectrometry with the EasyPep Mini MS Sample Prep

741 Kit (ThermoFisher, A40006) following the manufacturer's instructions. Briefly, proteins were

742 extracted, reduced, alkylated, and digested with trypsin/Lys-C protease. Hydrophilic and

743 hydrophobic contaminants were removed with a peptide clean-up step. LC-MS/MS analysis of

744 peptide samples was performed on an Ultimate 3000 rapid separation liquid chromatography

745 (RSLC) system connected to a Q-Exactive plus mass spectrometer (ThermoFisher). The

746 acquired raw files were processed with MaxQuant v1.6.6.0.

747 Western blotting. The cell surface biotinylated protein samples purified from HEK293T cells as

748 described above were heated to $95^{\circ} \mathrm{C}$ in SDS sample buffer for $10 \mathrm{~min} .15 \mu$ of each sample

749 was run on a polyacrylamide gel. Gels were blotted onto PVDF membranes (Merck). Blots were

750 blocked in 5\% milk in PBS, 0.2\% Tween-20 and incubated overnight with anti-HA rabbit 
751 monoclonal antibody (Cell Signaling Technology, 3724, RRID:AB_1549585) diluted 1:1,000 in

752 blocking solution. Blots were imaged with the $800 \mathrm{~nm}$ channel of a LI-COR Odyssey fluorescent

753 scanner after incubation with anti-rabbit DyLight 800-conjugated secondary antibody (Cell

754 Signaling Technology, 5151, RRID:AB_10697505) at 1:5,000 dilution for 30 min at room

755 temperature.

756 RNA-Seq analysis. We analysed data published by Hawdon and colleagues (74).

757 ArrayExpress run accession numbers and corresponding life cycle stages were: SRR6359160,

758 L3; SRR6359161 and SRR6359163, L4; SRR6359164 and SRR6359165, Adult mixed pooled

759 worms; SRR6359162 and SRR6359166, Adult male pooled worms. Our mapping and counting

760 strategy was modified as recommended for repetitive genomic features (90). Reads were

761 mapped against the reference $A$. ceylanicum genome (91) with STAR v2.7.5a (92) with

762 parameters: "--outSAMtype BAM SortedByCoordinate --runMode alignReads --

763 outFilterMultimapNmax 1000 --outSAMmultNmax 1 --outFilterMismatchNmax 3 --

764 outMultimapperOrder Random --winAnchorMultimapNmax 1000 --alignEndsType EndToEnd --

765 alignIntronMax 1 --alignMatesGapMax 350". FeatureCounts v2.0.1 (93) with parameters “-M -F

766 GFF -s 0 -p -t exon -g gene_id" was used to count reads over a modified annotation file based

767 on the original annotation (91) and downloaded from Wormbase Parasite (94). The original

768 annotation file in GFF format was slightly modified to contain a "gene_id" feature in the ninth

769 column and hence facilitate the calculation of aggregate reads by gene (for more details see

770 header of the GFF file, dataset S4). The resulting table of counts was imported into R (95) and

771 processed for differential expression analysis using DESeq2 v1.26.0 (96). Plots were generated

772 using ggplot2 (97) and karyoploteR (98). RNA-Seq analysis scripts and instructions for how to

773 use them are provided in the Supplementary Materials (dataset S5) and are additionally

774 available on Github [https://github.com/annaprotasio/Merchant_et_al_2020]. 
775 Statistics. Error bars represent the standard deviation or standard error -as indicated in the

776 respective figure legend- of 2-7 replicates conducted across at least two independent

777 experiments. SDS-PAGE gels and DLS data shown are representative of at least two

778 independent experiments. Significance and $P$-values were determined by 2-way ANOVA. For

779 DLS data, Sidak's multiple comparisons test was used with a 95\% confidence interval, in Prism

7808 (GraphPad). For cell-cell fusion data, Tukey's multiple comparisons test was used in Prism 9

781 (GraphPad). Source data are provided in datasets S1 and S2. No statistical methods were

782 used to predetermine sample size, experiments were not randomized, and the investigators

783 were not blinded to experimental outcomes.

\section{Data availability}

785 All data needed to evaluate the conclusions in the paper are present in the paper and/or the

786 Supplementary Materials. The atomic coordinates were deposited in the Protein Data Bank with

787 accession code 7A4A [https://doi.org/10.2210/pdb7A4A/pdb]. The cryo-EM density was

788 deposited in the EM Data Bank with code EMD-11630

789 [https://www.ebi.ac.uk/pdbe/entry/emdb/EMD-11630]. The raw electron micrographs were

790 deposited in EMPIAR with code EMPIAR-10266 [https://doi.org/10.6019/EMPIAR-10266]. RNA-

791 Seq analysis scripts and instructions for how to use them are provided in the Supplementary

792 Materials (dataset S5) and are additionally available on Github

793 [https://github.com/annaprotasio/Merchant_et_al_2020].

\section{References}

795 1. J. A. Frank, C. Feschotte, Co-option of endogenous viral sequences for host cell function.

$796 \quad$ Curr Opin Virol 25, 81-89 (2017).

797 2. A. Katzourakis, R. J. Gifford, Endogenous viral elements in animal genomes. PLoS genetics

798 6, e1001191 (2010). 
799 3. M. Friedli, D. Trono, The developmental control of transposable elements and the evolution 800 of higher species. Annu Rev Cell Dev Biol 31, 429-451 (2015).

801 4. E. B. Chuong, N. C. Elde, C. Feschotte, Regulatory evolution of innate immunity through 802 co-option of endogenous retroviruses. Science 351, 1083-1087 (2016).

803 5. A. Dupressoir, C. Lavialle, T. Heidmann, From ancestral infectious retroviruses to bona fide 804 cellular genes: role of the captured syncytins in placentation. Placenta 33, 663-671 (2012).

805 6. R. S. Moore et al., The role of the Cer1 transposon in horizontal transfer of

806 transgenerational memory. Cell 184, 4697-4712 e4618 (2021).

807 7. T. Gojobori, S. Yokoyama, Rates of evolution of the retroviral oncogene of Moloney murine 808 sarcoma virus and of its cellular homologues. Proc Natl Acad Sci U S A 82, 4198-4201 809 (1985).

810 8. K. R. McCarthy et al., The ancient fusogen EnvP(b)1 is expressed in human tissues and its 811 structure informs the evolution of gammaretrovirus envelope proteins. bioRxiv, $812 \quad 2020.2004 .2022 .056234(2020)$.

813 9. D. Kremer et al., Human endogenous retrovirus type W envelope protein inhibits 814 oligodendroglial precursor cell differentiation. Ann Neuro/ 74, 721-732 (2013).

815 10. W. Li et al., Human endogenous retrovirus-K contributes to motor neuron disease. Sci $816 \quad$ Transl Med 7, 307ra153 (2015).

817 11. M. Krupovic et al., Ortervirales: New Virus Order Unifying Five Families of Reverse$818 \quad$ Transcribing Viruses. J Virol 92, (2018).

819 12. I. G. Frame, J. F. Cutfield, R. T. Poulter, New BEL-like LTR-retrotransposons in Fugu 820 rubripes, Caenorhabditis elegans, and Drosophila melanogaster. Gene 263, 219-230 $821 \quad$ (2001).

822 13. N. J. Bowen, J. F. McDonald, Genomic analysis of Caenorhabditis elegans reveals ancient 823 families of retroviral-like elements. Genome Res 9, 924-935 (1999). 
824 14. H. S. Malik, S. Henikoff, T. H. Eickbush, Poised for contagion: evolutionary origins of the 825 infectious abilities of invertebrate retroviruses. Genome Res 10, 1307-1318 (2000).

826 15. D. Fass, S. C. Harrison, P. S. Kim, Retrovirus envelope domain at 1.7 angstrom resolution. $827 \quad$ Nat Struct Biol 3, 465-469 (1996).

828 16. D. C. Chan, D. Fass, J. M. Berger, P. S. Kim, Core structure of gp41 from the HIV envelope 829 glycoprotein. Cell 89, 263-273 (1997).

830 17. W. Weissenhorn, A. Dessen, S. C. Harrison, J. J. Skehel, D. C. Wiley, Atomic structure of 831 the ectodomain from HIV-1 gp41. Nature 387, 426-430 (1997).

832 18. B. Kobe, R. J. Center, B. E. Kemp, P. Poumbourios, Crystal structure of human T cell 833 leukemia virus type $1 \mathrm{gp} 21$ ectodomain crystallized as a maltose-binding protein chimera 834 reveals structural evolution of retroviral transmembrane proteins. Proc Natl Acad Sci U S A $83596,4319-4324$ (1999).

836 19. S. C. Harrison, Viral membrane fusion. Virology 479-480, 498-507 (2015).

837 20. M. Dessau, Y. Modis, Crystal structure of glycoprotein C from Rift Valley fever virus. Proc. $838 \quad$ Natl. Acad. Sci. U. S. A. 110, 1696-1701 (2013).

839 21. Y. Modis, Relating Structure to Evolution in Class II Viral Membrane Fusion Proteins. Curr $840 \quad$ Opin Virol, DOI:10.1016/j.coviro.2014.1001.1009 (2014).

841 22. J. Lescar et al., The Fusion glycoprotein shell of Semliki Forest virus: an icosahedral 842 assembly primed for fusogenic activation at endosomal pH. Cell 105, 137-148 (2001).

843 23. F. A. Rey, F. X. Heinz, C. Mandl, C. Kunz, S. C. Harrison, The envelope glycoprotein from 844 tick-borne encephalitis virus at 2 A resolution. Nature 375, 291-298 (1995).

845 24. Y. Modis, S. Ogata, D. Clements, S. C. Harrison, A ligand-binding pocket in the dengue 846 virus envelope glycoprotein. Proc Natl Acad Sci U S A 100, 6986-6991 (2003).

847 25. R. M. DuBois et al., Functional and evolutionary insight from the crystal structure of rubella $848 \quad$ virus protein E1. Nature 493, 552-556 (2013). 
849 26. Y. Modis, S. Ogata, D. Clements, S. C. Harrison, Structure of the dengue virus envelope $850 \quad$ protein after membrane fusion. Nature 427, 313-319 (2004).

851 27. D. L. Gibbons et al., Conformational change and protein-protein interactions of the fusion 852 protein of Semliki Forest virus. Nature 427, 320-325 (2004).

853 28. P. Guardado-Calvo et al., A glycerophospholipid-specific pocket in the RVFV class II fusion 854 protein drives target membrane insertion. Science 358, 663-667 (2017).

855 29. J. Perez-Vargas et al., Structural basis of eukaryotic cell-cell fusion. Cell 157, 407-419 $856 \quad$ (2014).

857 30. O. Avinoam et al., Conserved eukaryotic fusogens can fuse viral envelopes to cells.

$858 \quad$ Science 332, 589-592 (2011).

859 31. A. Sapir et al., AFF-1, a FOS-1-regulated fusogen, mediates fusion of the anchor cell in C. $860 \quad$ elegans. Dev Cell 12, 683-698 (2007).

861 32. B. Podbilewicz et al., The C. elegans developmental fusogen EFF-1 mediates homotypic 862 fusion in heterologous cells and in vivo. Dev Cell 11, 471-481 (2006).

863 33. J. Fedry et al., The Ancient Gamete Fusogen HAP2 Is a Eukaryotic Class II Fusion Protein. $864 \quad$ Cell 168, 904-915 e910 (2017).

865 34. J. Fedry et al., Evolutionary diversification of the HAP2 membrane insertion motifs to drive 866 gamete fusion across eukaryotes. PLoS Biol 16, e2006357 (2018).

867 35. J. Feng et al., Fusion surface structure, function, and dynamics of gamete fusogen HAP2. 868 elife 7, (2018).

869 36. C. Valansi et al., Arabidopsis HAP2/GCS1 is a gamete fusion protein homologous to 870 somatic and viral fusogens. J Cell Biol 216, 571-581 (2017).

871 37. T. Mori, H. Kuroiwa, T. Higashiyama, T. Kuroiwa, GENERATIVE CELL SPECIFIC 1 is 872 essential for angiosperm fertilization. Nat Cell Biol 8, 64-71 (2006). 
873 38. K. von Besser, A. C. Frank, M. A. Johnson, D. Preuss, Arabidopsis HAP2 (GCS1) is a 874 sperm-specific gene required for pollen tube guidance and fertilization. Development 133, $875 \quad 4761-4769(2006)$.

876 39. T. Clark, HAP2/GCS1: Mounting evidence of our true biological EVE? PLoS Biol 16, 877 e3000007 (2018).

878 40. S. F. Altschul et al., Gapped BLAST and PSI-BLAST: a new generation of protein database 879 search programs. Nucleic Acids Res 25, 3389-3402 (1997).

880 41. O. Poch, I. Sauvaget, M. Delarue, N. Tordo, Identification of four conserved motifs among 881 the RNA-dependent polymerase encoding elements. EMBO J 8, 3867-3874 (1989).

882 42. H. Felder et al., Tas, a retrotransposon from the parasitic nematode Ascaris lumbricoides. 883 Gene 149, 219-225 (1994).

884 43. M. Krupovic, E. V. Koonin, Homologous Capsid Proteins Testify to the Common Ancestry of 885 Retroviruses, Caulimoviruses, Pseudoviruses, and Metaviruses. J Virol 91, (2017).

886 44. I. Letunic, P. Bork, Interactive Tree Of Life (iTOL) v4: recent updates and new developments. Nucleic Acids Res 47, W256-W259 (2019).

888 45. S. Halldorsson et al., Structure of a phleboviral envelope glycoprotein reveals a 889 consolidated model of membrane fusion. Proc Natl Acad Sci U S A 113, 7154-7159 (2016).

890 46. Y. Zhu et al., The Postfusion Structure of the Heartland Virus Gc Glycoprotein Supports 891 Taxonomic Separation of the Bunyaviral Families Phenuiviridae and Hantaviridae. J Virol 892 92, (2018).

893 47. D. J. Leahy, W. A. Hendrickson, I. Aukhil, H. P. Erickson, Structure of a fibronectin type III 894 domain from tenascin phased by MAD analysis of the selenomethionyl protein. Science $895 \quad$ 258, 987-991 (1992).

896 48. J. F. Pinello et al., Structure-Function Studies Link Class II Viral Fusogens with the 897 Ancestral Gamete Fusion Protein HAP2. Curr Biol 27, 651-660 (2017). 
49. M. Kielian, F. A. Rey, Virus membrane-fusion proteins: more than one way to make a hairpin. Nat. Rev. Microbiol. 4, 67-76 (2006).

900 50. L. Holm, P. Rosenstrom, Dali server: conservation mapping in 3D. Nucleic Acids Res 38, 901 W545-549 (2010).

902 51. D. E. Klein, J. L. Choi, S. C. Harrison, Structure of a dengue virus envelope protein late-

903 stage fusion intermediate. J Virol 87, 2287-2293 (2013).

904 52. S. Murakami, K. Terasaki, S. I. Ramirez, J. C. Morrill, S. Makino, Development of a novel, 905 single-cycle replicable rift valley Fever vaccine. PLoS neglected tropical diseases 8, e2746 906 (2014).

907 53. E. Zaitseva, S. T. Yang, K. Melikov, S. Pourmal, L. V. Chernomordik, Dengue virus ensures 908 its fusion in late endosomes using compartment-specific lipids. PLOS Pathog. 6, e1001131 909 (2010).

910 54. P. Y. Lozach et al., Entry of bunyaviruses into mammalian cells. Cell Host Microbe 7, 488$911499(2010)$.

912 55. A. M. Nour, Y. Li, J. Wolenski, Y. Modis, Viral membrane fusion and nucleocapsid delivery 913 into the cytoplasm are distinct events in some flaviviruses. PLoS Pathog 9, e1003585 914 (2013).

915 56. Y. Kubo, H. Hayashi, T. Matsuyama, H. Sato, N. Yamamoto, Retrovirus entry by 916 endocytosis and cathepsin proteases. Adv Virol 2012, 640894 (2012).

917 57. D. Bitto, S. Halldorsson, A. Caputo, J. T. Huiskonen, Low pH and Anionic Lipid-dependent 918 Fusion of Uukuniemi Phlebovirus to Liposomes. J Biol Chem 291, 6412-6422 (2016).

919 58. D. L. Esposito, J. B. Nguyen, D. C. DeWitt, E. Rhoades, Y. Modis, Physico-chemical 920 requirements and kinetics of membrane fusion of flavivirus-like particles. J Gen Virol 96, $921 \quad 1702-1711(2015)$.

922 59. M. Umashankar et al., Differential cholesterol binding by class II fusion proteins determines 923 membrane fusion properties. J Virol 82, 9245-9253 (2008). 
924 60. P. K. Chatterjee, C. H. Eng, M. Kielian, Novel mutations that control the sphingolipid and 925 cholesterol dependence of the Semliki Forest virus fusion protein. J Virol 76, 12712-12722 $926 \quad$ (2002).

927 61. J. M. Smit, R. Bittman, J. Wilschut, Low-pH-dependent fusion of Sindbis virus with receptor928 free cholesterol- and sphingolipid-containing liposomes. J Virol 73, 8476-8484 (1999).

929 62. T. V. Kurzchalia, S. Ward, Why do worms need cholesterol? Nat Cell Biol 5, 684-688 $930 \quad$ (2003).

931 63. M. Merris, J. Kraeft, G. S. Tint, J. Lenard, Long-term effects of sterol depletion in C. 932 elegans: sterol content of synchronized wild-type and mutant populations. J Lipid Res 45, 933 2044-2051 (2004).

934 64. M. Ruiz et al., Membrane fluidity is regulated by the C. elegans transmembrane protein 935 FLD-1 and its human homologs TLCD1/2. eLife 7, (2018).

936 65. K. Stiasny, S. L. Allison, J. Schalich, F. X. Heinz, Membrane interactions of the tick-borne encephalitis virus fusion protein E at low pH. J. Virol. 76, 3784-3790 (2002).

938 66. D. L. Gibbons, A. Ahn, P. K. Chatterjee, M. Kielian, Formation and characterization of the 939 trimeric form of the fusion protein of Semliki Forest Virus. J Virol 74, 7772-7780 (2000).

940 67. R. Fritz, K. Stiasny, F. X. Heinz, Identification of specific histidines as $\mathrm{pH}$ sensors in 941 flavivirus membrane fusion. J Cell Biol 183, 353-361 (2008).

942 68. V. Nayak et al., Crystal structure of dengue virus type 1 envelope protein in the postfusion 943 conformation and its implications for membrane fusion. J. Virol. 83, 4338-4344 (2009).

944 69. Z. L. Qin, Y. Zheng, M. Kielian, Role of conserved histidine residues in the low-pH 945 dependence of the Semliki Forest virus fusion protein. J Virol 83, 4670-4677 (2009).

946 70. Y. Zheng, C. Sanchez-San Martin, Z. L. Qin, M. Kielian, The domain I-domain III linker 947 plays an important role in the fusogenic conformational change of the alphavirus membrane 948 fusion protein. J Virol 85, 6334-6342 (2011). 
949 71. S. M. de Boer et al., Acid-activated structural reorganization of the Rift Valley fever virus Gc 950 fusion protein. J Virol 86, 13642-13652 (2012).

951 72. M. A. Vega, J. L. Strominger, Constitutive endocytosis of HLA class I antigens requires a

952 specific portion of the intracytoplasmic tail that shares structural features with other

953 endocytosed molecules. Proc Natl Acad Sci U S A 86, 2688-2692 (1989).

954 73. R. DeMarco et al., Saci-1, -2, and -3 and Perere, four novel retrotransposons with high

955 transcriptional activities from the human parasite Schistosoma mansoni. J Virol 78, 2967-

$2956(2004)$.

957 74. J. P. Bernot et al., Transcriptomic analysis of hookworm Ancylostoma ceylanicum life cycle

958 stages reveals changes in G-protein coupled receptor diversity associated with the onset of

959 parasitism. Int J Parasitol 50, 603-610 (2020).

960 75. D. Moi et al., Archaeal origins of gamete fusion. bioRxiv, 2021.2010.2013.464100 (2021).

961 76. R. S. Moore et al., Horizontal and vertical transmission of transgenerational memories via

962 the Cer1 transposon. bioRxiv, 2020.2012.2028.424563 (2020).

963 77. S. Kurtz et al., REPuter: the manifold applications of repeat analysis on a genomic scale.

$964 \quad$ Nucleic Acids Res 29, 4633-4642 (2001).

965 78. B. M. Muhire, A. Varsani, D. P. Martin, SDT: a virus classification tool based on pairwise 966 sequence alignment and identity calculation. PLoS One 9, e108277 (2014).

967 79. S. Q. Zheng et al., MotionCor2: anisotropic correction of beam-induced motion for improved $968 \quad$ cryo-electron microscopy. Nat Methods 14, 331-332 (2017).

969 80. K. Zhang, Gctf: Real-time CTF determination and correction. J Struct Biol 193, 1-12 (2016).

970 81. S. He, S. H. W. Scheres, Helical reconstruction in RELION. J Struct Biol 198, 163-176

$971 \quad$ (2017).

972 82. S. H. Scheres, RELION: implementation of a Bayesian approach to cryo-EM structure

973 determination. J Struct Biol 180, 519-530 (2012). 
974 83. J. Zivanov, T. Nakane, S. H. W. Scheres, A Bayesian approach to beam-induced motion 975 correction in cryo-EM single-particle analysis. IUCrJ 6, 5-17 (2019).

976 84. E. F. Pettersen et al., UCSF Chimera--a visualization system for exploratory research and 977 analysis. J Comput Chem 25, 1605-1612 (2004).

978 85. P. D. Adams et al., PHENIX: a comprehensive Python-based system for macromolecular 979 structure solution. Acta Crystallogr D Biol Crystallogr 66, 213-221 (2010).

980 86. P. Emsley, K. Cowtan, Coot: model-building tools for molecular graphics. Acta Crystallogr D 981 Biol Crystallogr 60, 2126-2132 (2004).

982 87. A. Amunts et al., Structure of the yeast mitochondrial large ribosomal subunit. Science 343, 983 1485-1489 (2014).

984 88. V. B. Chen et al., MolProbity: all-atom structure validation for macromolecular 985 crystallography. Acta Crystallogr D Biol Crystallogr 66, 12-21 (2010).

986 89. J. Schindelin et al., Fiji: an open-source platform for biological-image analysis. Nat Methods 9879 9, 676-682 (2012).

988 90. A. Teissandier, N. Servant, E. Barillot, D. Bourc'his, Tools and best practices for 989 retrotransposon analysis using high-throughput sequencing data. Mob DNA 10, 52 (2019).

990 91. E. M. Schwarz et al., The genome and transcriptome of the zoonotic hookworm

991 Ancylostoma ceylanicum identify infection-specific gene families. Nat Genet 47, 416-422 992 (2015).

993 92. A. Dobin, T. R. Gingeras, Mapping RNA-seq Reads with STAR. Curr Protoc Bioinformatics $994 \quad 51,11.14 .11-11.14 .19(2015)$.

995 93. Y. Liao, G. K. Smyth, W. Shi, featureCounts: an efficient general purpose program for 996 assigning sequence reads to genomic features. Bioinformatics 30, 923-930 (2014).

997 94. K. L. Howe, B. J. Bolt, M. Shafie, P. Kersey, M. Berriman, WormBase ParaSite - a

998 comprehensive resource for helminth genomics. Molecular and biochemical parasitology $999215,2-10(2017)$. 
1000 95. R. C. Team. (R Foundation for Statistical Computing, Vienna, Austria, 2019).

1001 96. M. I. Love, W. Huber, S. Anders, Moderated estimation of fold change and dispersion for 1002 RNA-seq data with DESeq2. Genome Biol 15, 550 (2014).

1003 97. H. Wickham, ggplot2: Elegant Graphics for Data Analysis. (Springer-Verlag, New York, 1004 2016).

1005 98. B. Gel, E. Serra, karyoploteR: an R/Bioconductor package to plot customizable genomes 1006 displaying arbitrary data. Bioinformatics 33, 3088-3090 (2017).

\section{Acknowledgements}

1008 We thank Sew-Yeu Peak-Chew at the MRC Laboratory of Molecular Biology (LMB) Mass

1009 Spectrometry Facility for assistance with mass spectrometry data collection and analysis. We 1010 acknowledge the MRC-LMB Electron Microscopy (EM) Facility and UK national electron Bio-

1011 Imaging Centre (eBIC) at Diamond Light Source (DLS) for access and support of EM sample

1012 preparation and data collection. We acknowledge DLS for access and support of eBIC under

1013 proposals EM17434 and EM20121 funded by the Wellcome Trust, MRC and BBSRC. We are

1014 grateful to Kyle Dent for aiding in using the microscopes at eBIC. We are grateful for access to

1015 the MRC-LMB Scientific Computing facilities. This work was supported by Senior Research

1016 Fellowships 101908/Z/13/Z and 217191/Z/19/Z from the Wellcome Trust and NIH grant R01

1017 GM102869-01 to Y.M.

\section{Author contributions}

$1019 \mathrm{MM}$ and YM conceived the experiments. MM purified the proteins and performed the

1020 biochemical assays. CPM collected and processed the cryo-EM data and performed the image

1021 reconstruction. CPM and YM built and refined the atomic model. YL and HZ carried out the cell-

1022 cell fusion assay. AVP performed the RNA-Seq analysis. All authors contributed to the figures.

1023 YM wrote the manuscript. Project supervision, administration, and funding acquisition, YM.

\section{Competing interests}


1025 YM is a consultant for Related Sciences LLC and has profits interests in Danger Bio LLC. The

1026 authors declare no other competing interests.

1027 Materials \& Correspondence

1028 Correspondence and material requests should be addressed to Y.M. (ymodis@mrc-

1029 Imb.cam.ac.uk). 
1032 Monique Merchant, Carlos P. Mata, Yangci Liu, Haoming Zhai, Anna V. Protasio,

\author{
Yorgo Modis*
}

1034

*Corresponding author. Email: ymodis@mrc-Imb.cam.ac.uk

1035

1036

Supplementary Materials

Figures S1 to S10

Movie S1 

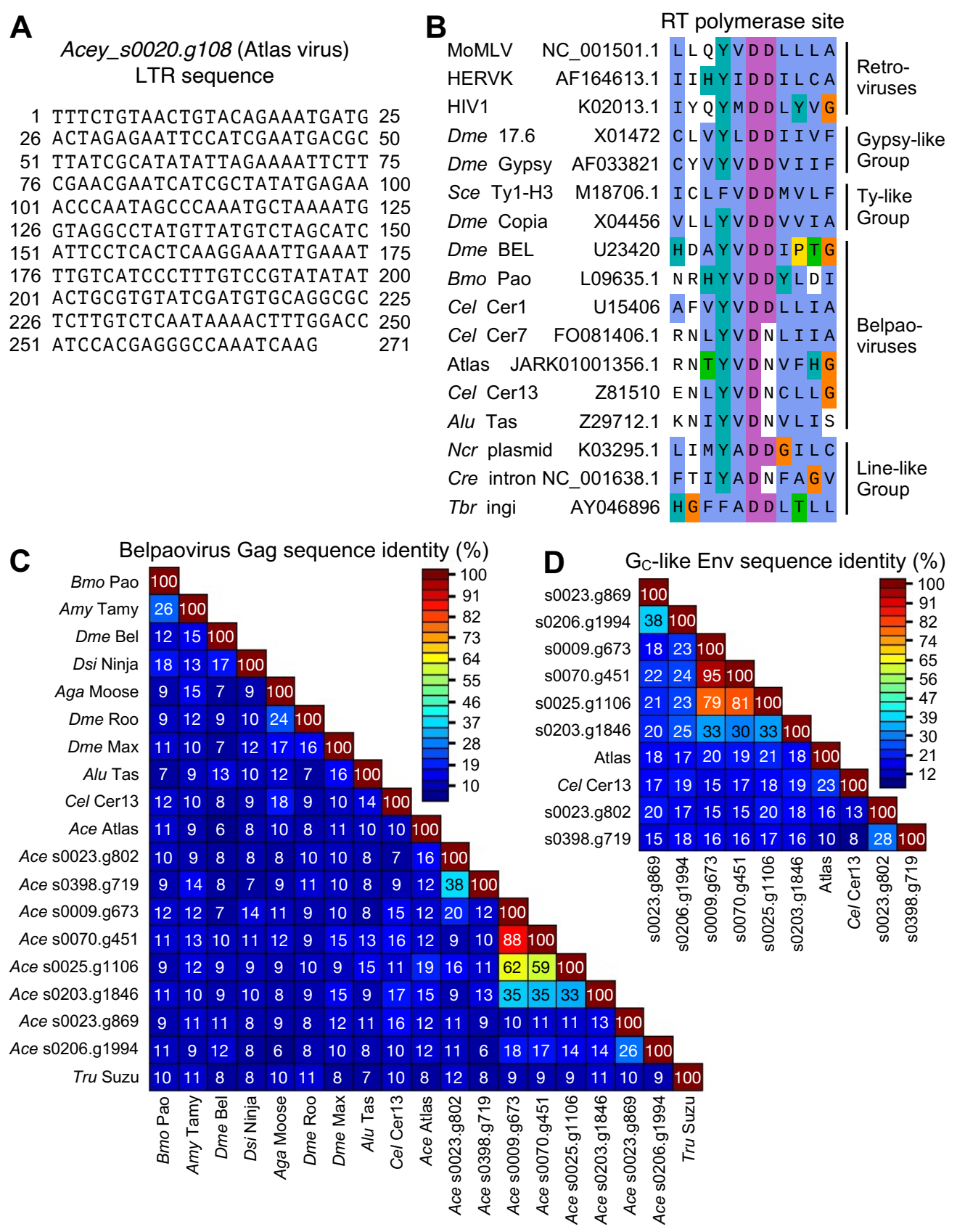

Fig. S1. Atlas is an endogenous belpaovirus with a phlebovirus-like Env. (A) Nucleotide sequence of the Atlas LTRs, predicted by REPuter (76). The LTRs are $100 \%$ identical. (B) Aligned protein sequences of reverse transcriptase (RT) polymerase site from Atlas and other representative belpao- and retroviruses. A. ceylanicum belpaoviruses have an Asp to Asn substitution (Y[X]DD to YVDN) in the polymerase site, the most conserved RT motif (Motif V, or Motif C). GenBank accession numbers are listed after virus names. MoMLV, Moloney murine leukemia virus; HERVK, human ERV-K; Dme, Drosophila melanogaster, Sce, Saccharomyces cerevisiae; Bmo, Bombyx mori; Cel, C. elegans; Ace, A. ceylanicum; Alu, Ascaris lumbricoides; Ncr, Neurospora crassa; Cre, C. reinhardtii; Tbr, Trypanosoma brucei. (C) Sequence identity matrix for Gag protein sequences from representative belpaoviruses and $A$. ceylanicum belpaoviruses with intact protein coding regions. Amy, Antheraea mylitta; Dsi, Drosophila simulans; Aga, Anopheles gambiae; Tru, Takifugu rubripes. (D) Sequence identity matrix for phlebovirus-like Env protein sequences from nematode 1056 belpaoviruses. Identity matrices were calculated with SDT (77). 

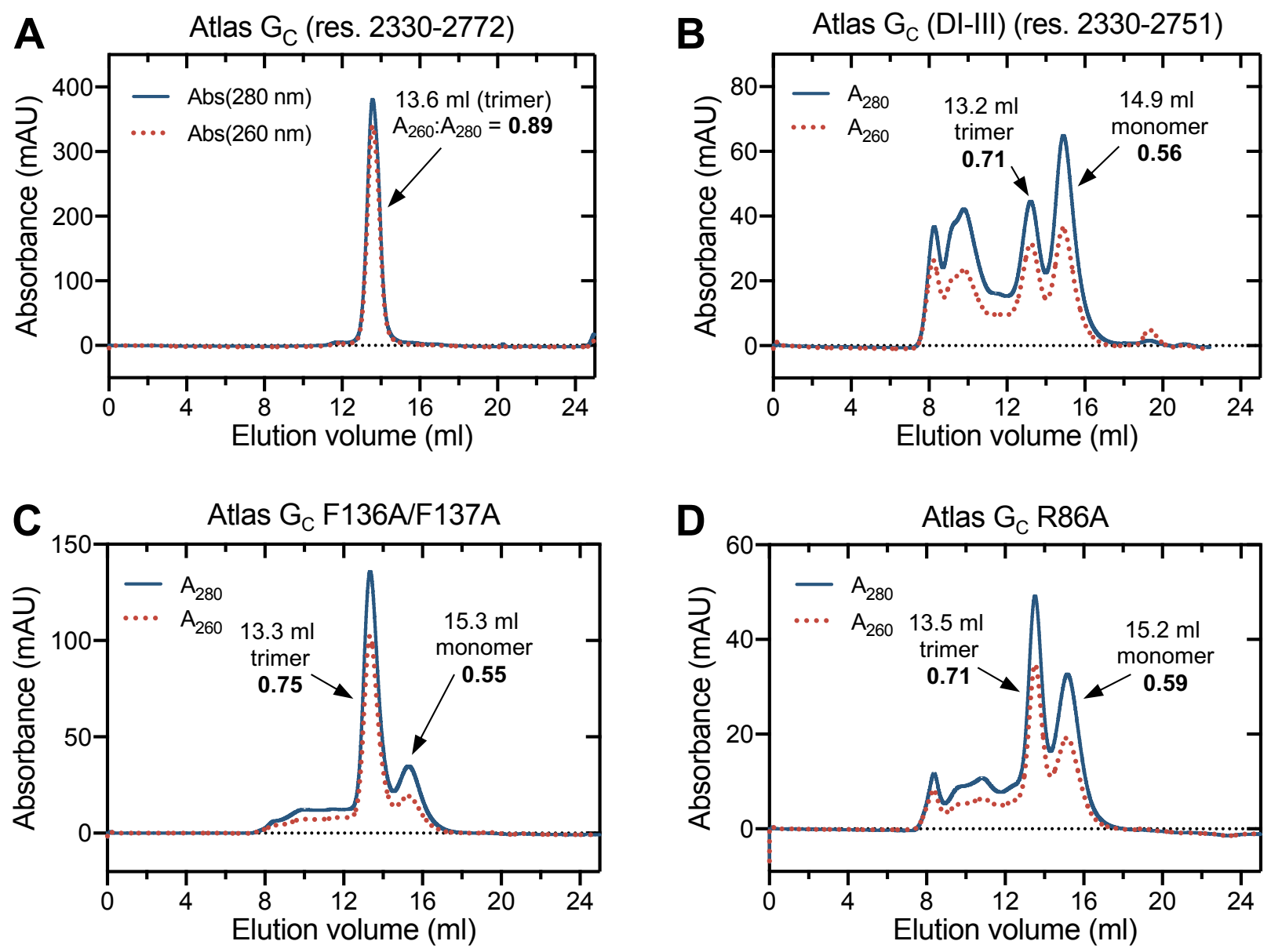

Fig. S2. Size-exclusion chromatography of Atlas $G_{c}$ following ion-exchange chromatography. Samples were analysed on a Superdex 200 Increase (10/300) column (Cytiva). The $\mathrm{A}_{260}: \mathrm{A}_{280}$ ratios for selected peaks are shown in bold. (A) The Atlas $\mathrm{G}_{\mathrm{C}}$ fragment used for cryo-EM image reconstruction (residues 2330-2772). The protein had higher absorbance at $260 \mathrm{~nm}\left(\mathrm{~A}_{260}\right)$ than expected for pure protein. The elution volume was consistent with a homotrimer. (B) Atlas $\mathrm{G}_{\mathrm{c}}(\mathrm{DI}-\mathrm{III})$, maintained at $\mathrm{pH}>7$ throughout expression and purification. Multiple oligomeric states were present including peaks with elution volumes consistent with monomers, trimers, and higher order oligomers. (C) Atlas $\mathrm{G}_{\mathrm{C}}$ fusion loop mutant F136A/F137A. (D) Atlas $G_{c}$ GPL binding pocket mutant R86A. 

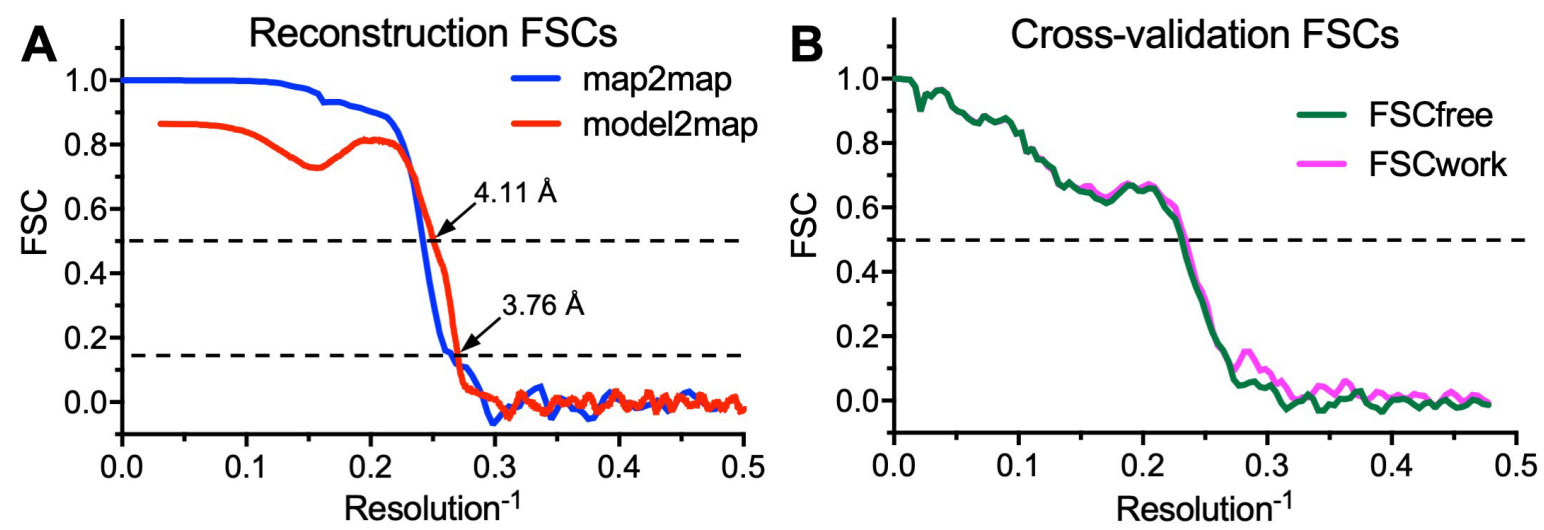

C 3,027 movies $\stackrel{\text { MotionCorr2, GCTF }}{\longrightarrow} 2,864$ averaged movies

$\downarrow$ Autopick (2D references from previous dataset)

987,570 particles

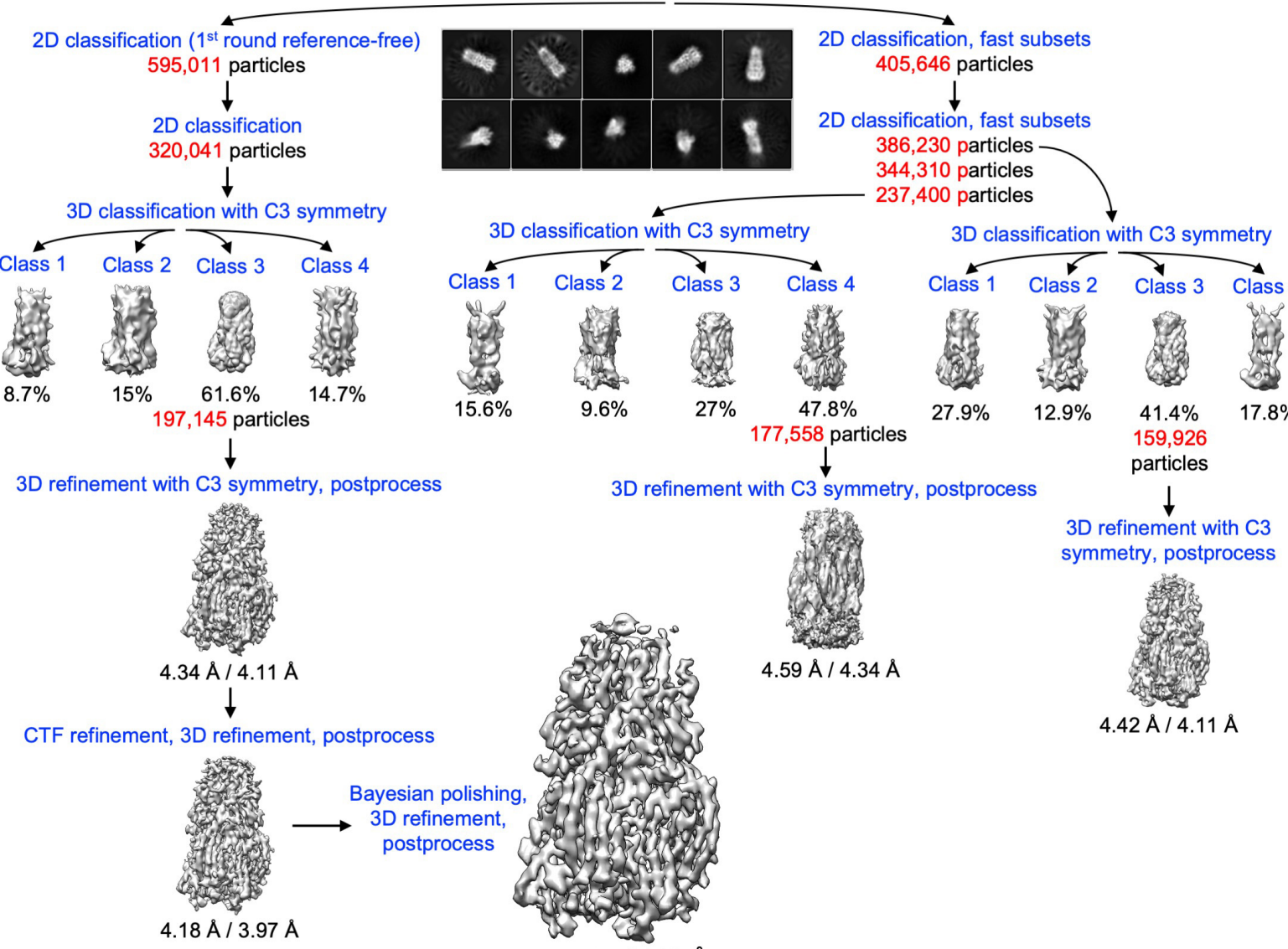

Fig. S3. Image reconstruction quality assessment and workflow. (A) Fourier Shell

Correlations (FSC) of the reconstruction of Atlas $\mathrm{Gc}_{\mathrm{c}}$ from two independently refined half-maps (map2map,

1070 in blue); and of the reconstruction from the whole dataset versus a map calculated from the refined atomic model (model2map, in red). The gold-standard cutoff $(F S C=0.143$ ) and the $F S C=0.5$ level are marked with dashed lines. The resolution values of each curve at these levels are indicated. (B) FSC plots for cross-validation as described by Amunts et al. (86). FSCwork (magenta), FSC of refined test model versus work set (half-map used in test refinement). FSCfree (green), FSC of refined test model versus test set (half-map not used in test refinement). The FSC $=0.5$ level is indicated by a dashed line. (C) Flow chart showing the workflow pipeline for cryo-EM image processing, classification, and model 1077 refinement, as described in the Materials and Methods. 

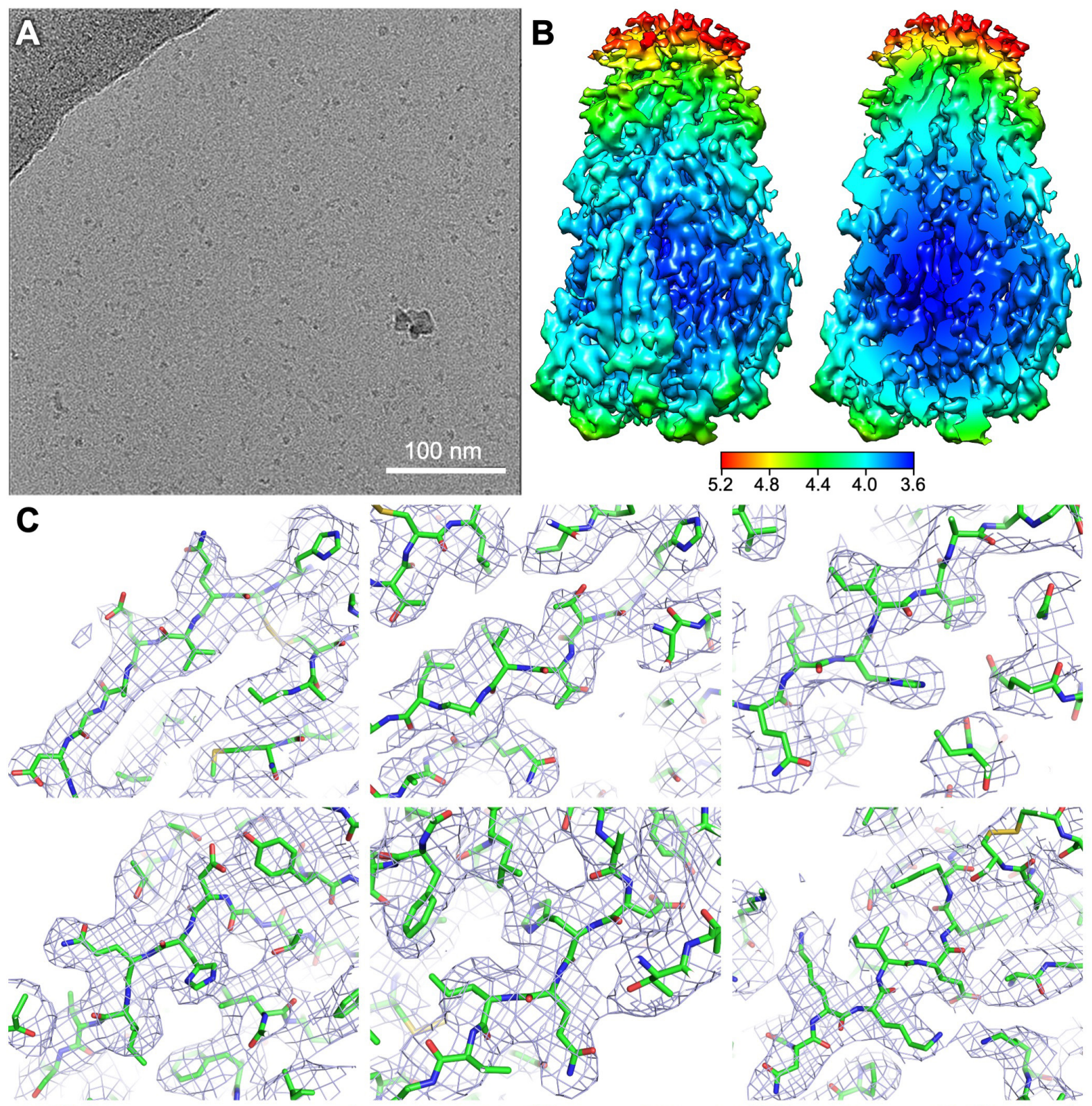

1078
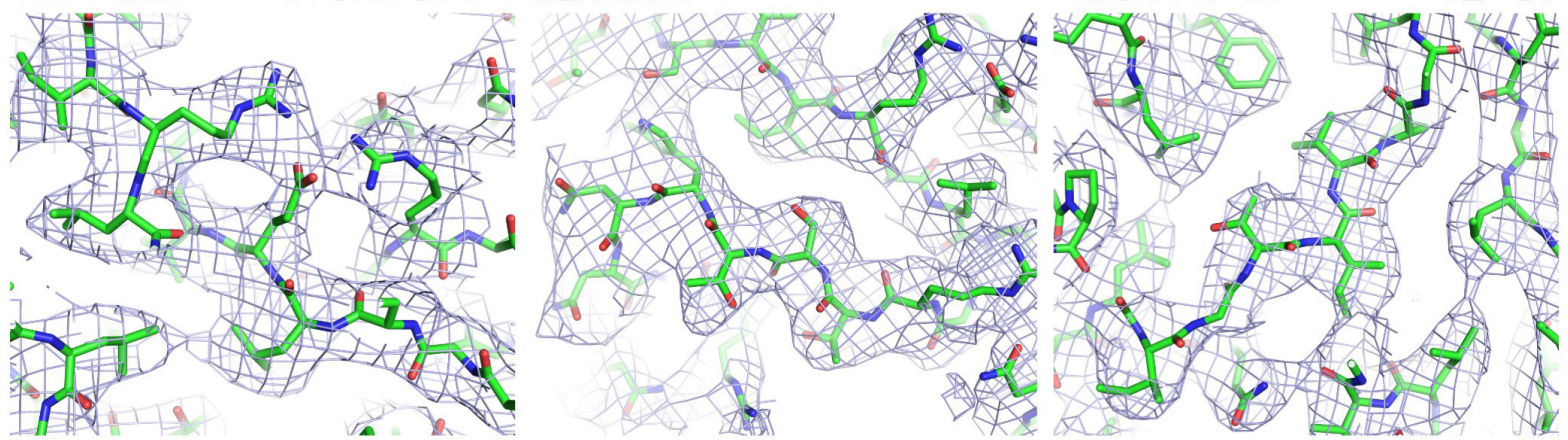

Fig. S4. Representative Cryo-EM micrograph and density with local resolution estimation (A) Representative cryo-EM micrograph of Atlas $\mathrm{G}_{\mathrm{c}}$ trimers. (B) Local resolution estimation for the cryo-EM volume, calculated in RELION 3.0 (82). (C) Representative samples of local cryoEM density from the structure with fitted and refined atomic models. The deposited density map 1083 was contoured at $3 \sigma$ in Pymol (Schrodinger, LLC). 


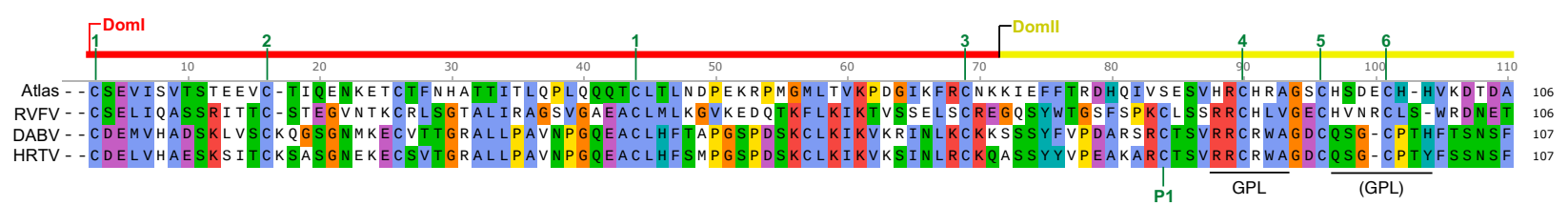

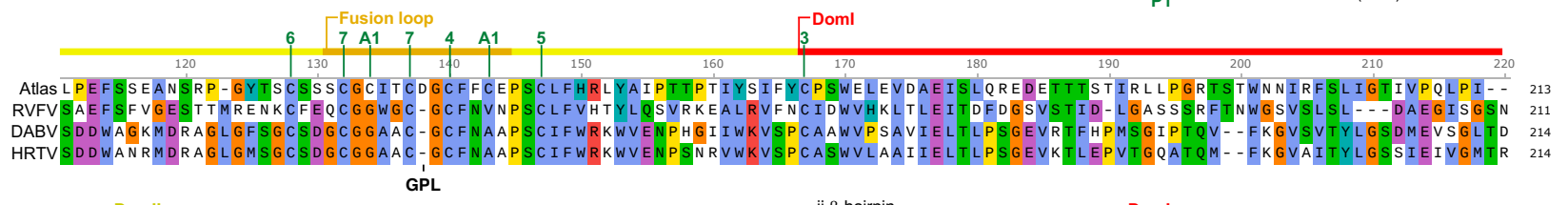

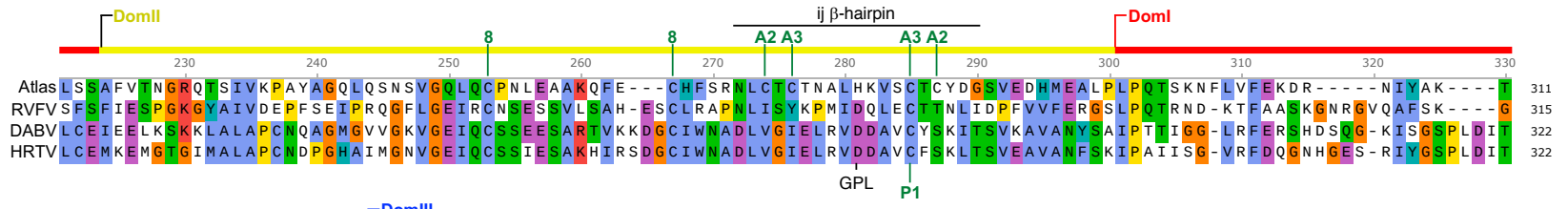

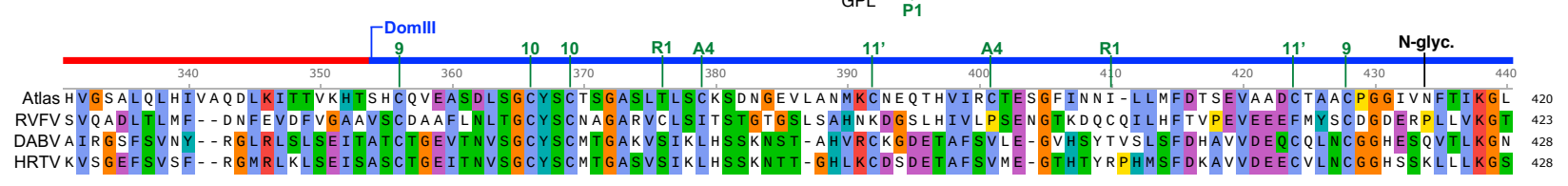

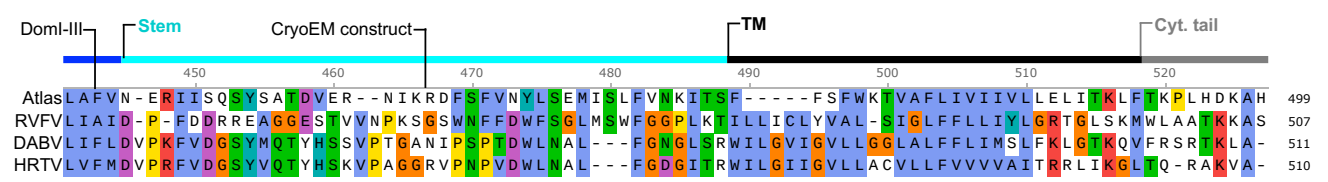

1085 Fig. S5. Protein sequence alignment of Atlas $\mathbf{G}_{\mathrm{c}}$ and its closest orthologs Amino acid sequence alignment of Atlas $G_{C}$ and the most similar sequences from infectious viruses: Rift Valley Fever virus (RVFV), Dabie bandavirus (DABV, formerly SFTSV phlebovirus) and Heartland virus (HRTV). Domains and structural features are marked with lines above the alignment. GPL, residues in or near the glycerophospholipid headgroup binding pocket. N-glyc., $\mathrm{N}$-glycosylation site. Cyt. tail, cytoplasmic tail. Conserved disulfide bonds are numbered in green. Disulfide bonds specific to Atlas virus are denoted with an "A". An RVFV-specific disulfide is denoted with an "R". A phlebovirus-specific disulfide is denoted with a "P". The 1094 disulfide marked 11' is conserved in Atlas, DABV and HRTV but not RVFV. 


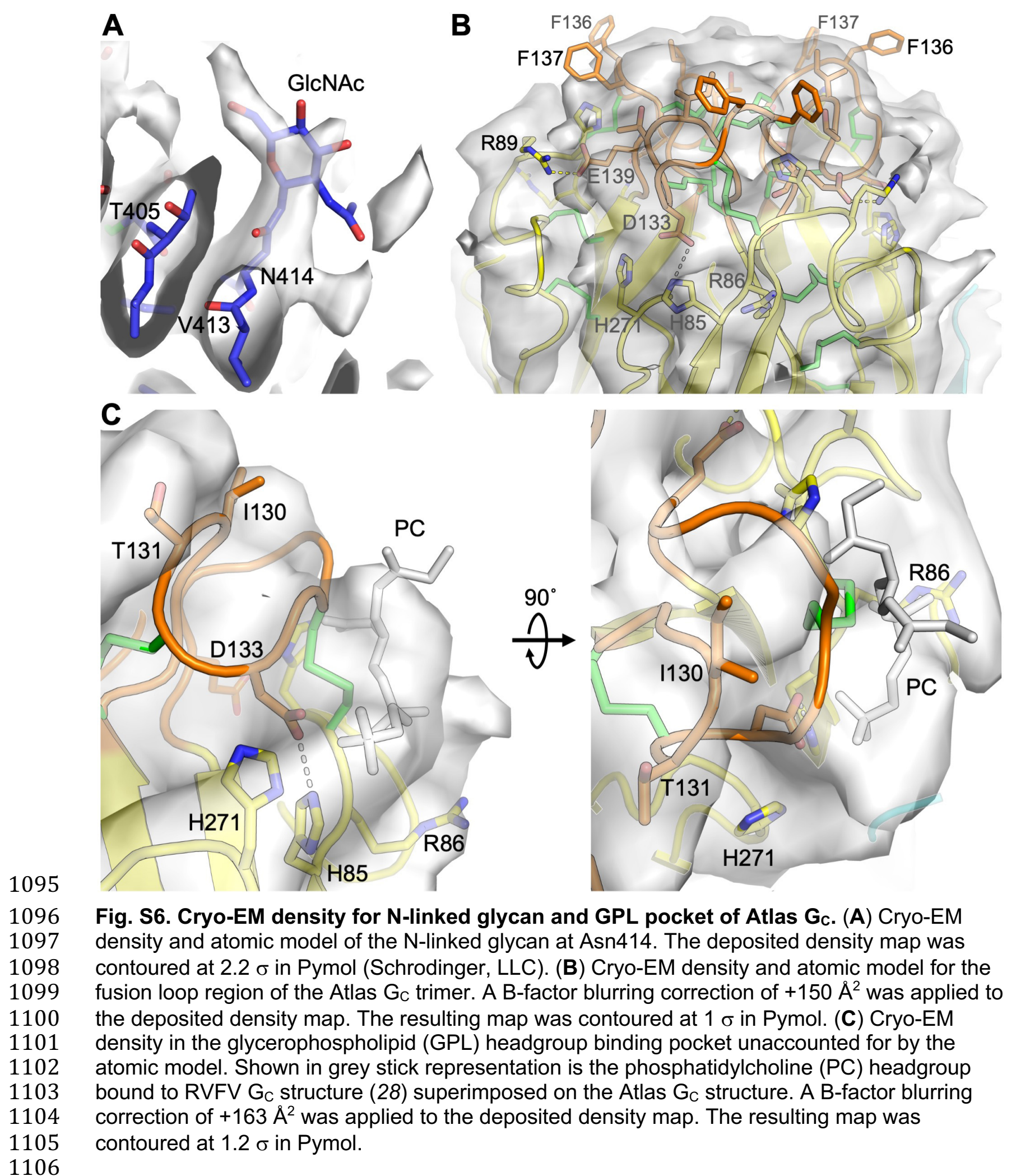



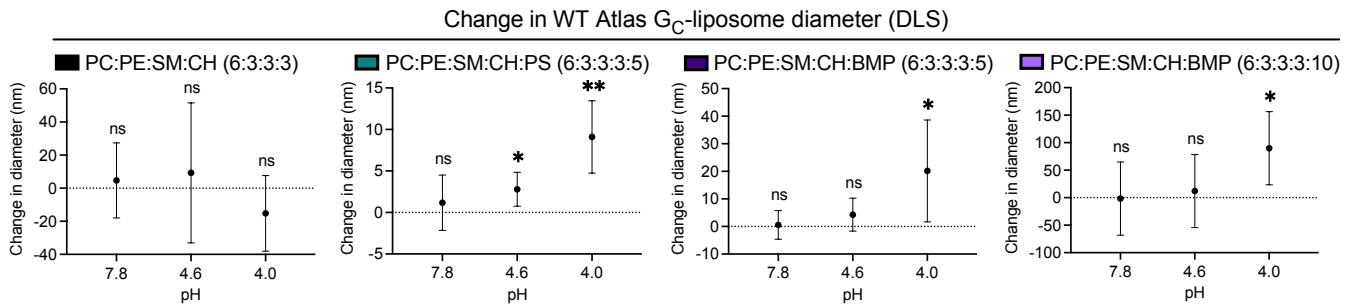

Change in $\mathrm{F} 136 \mathrm{~A} / \mathrm{F} 137 \mathrm{~A}$ Atlas $\mathrm{G}_{\mathrm{C}}$-liposome diameter (DLS)
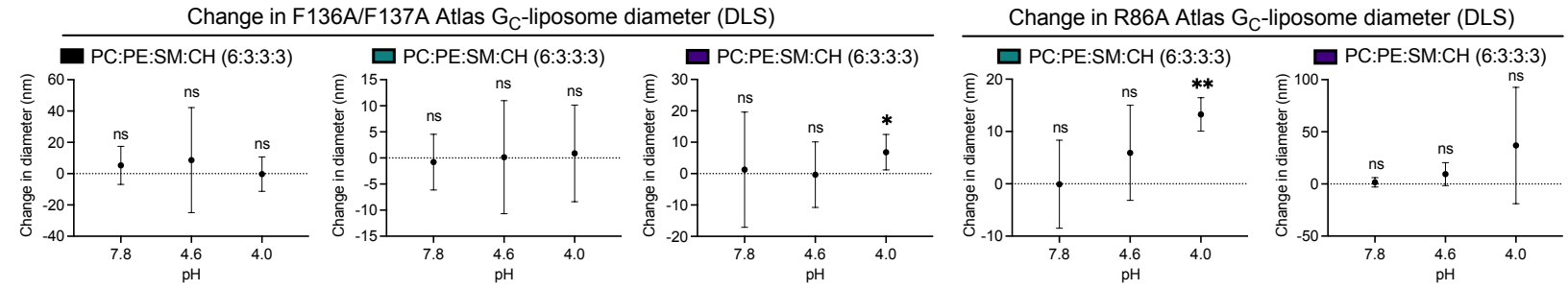

Change in WT Atlas $\mathrm{G}_{\mathrm{C}}$-liposome diameter (DLS)
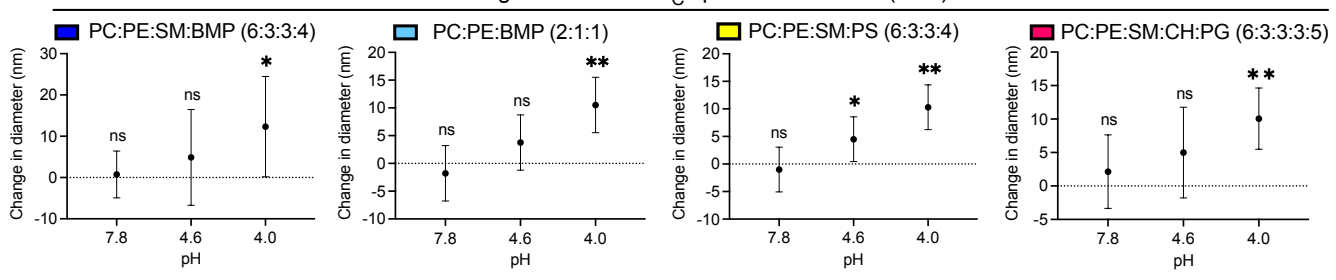

Fig. S7. Dynamic light scattering of Atlas $\mathbf{G}_{c}$-liposome complexes. Liposome diameter dynamic light scattering (DLS) data from Fig. 4B are shown as the mean change in liposome diameter upon binding Atlas $\mathrm{G}_{c}$ ectodomain (WT, F136A/F137A or R86A). Error bars show the standard error of the mean (s.e.m.) of three to seven replicates (see dataset $\mathbf{S 1}$ for source data). Significance was determined by 2-way ANOVA analysis using Sidak's multiple comparisons test with a 95\% confidence interval, in Prism 8 (GraphPad). ${ }^{* \star}, 0.001<p<0.01$; *, $1114 p<0.05$; ns, not significant. 
Fig. 4A

WT

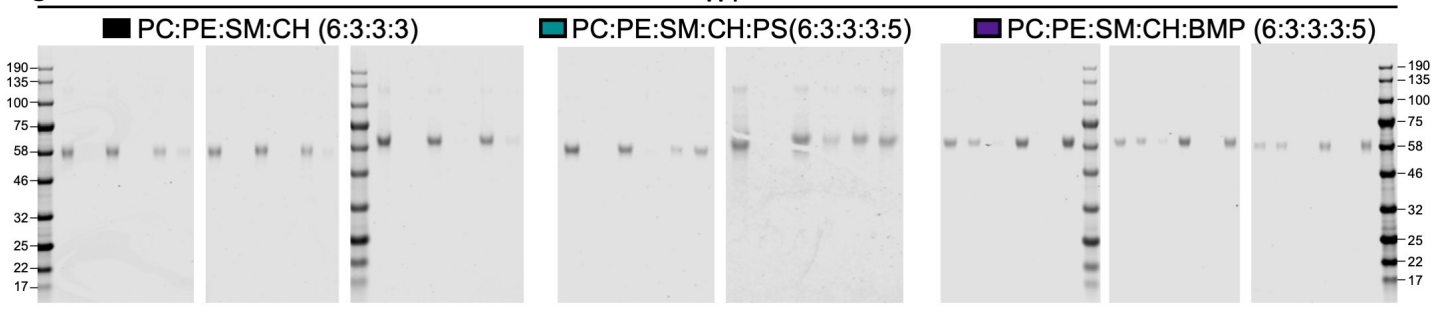

F136A/F137A
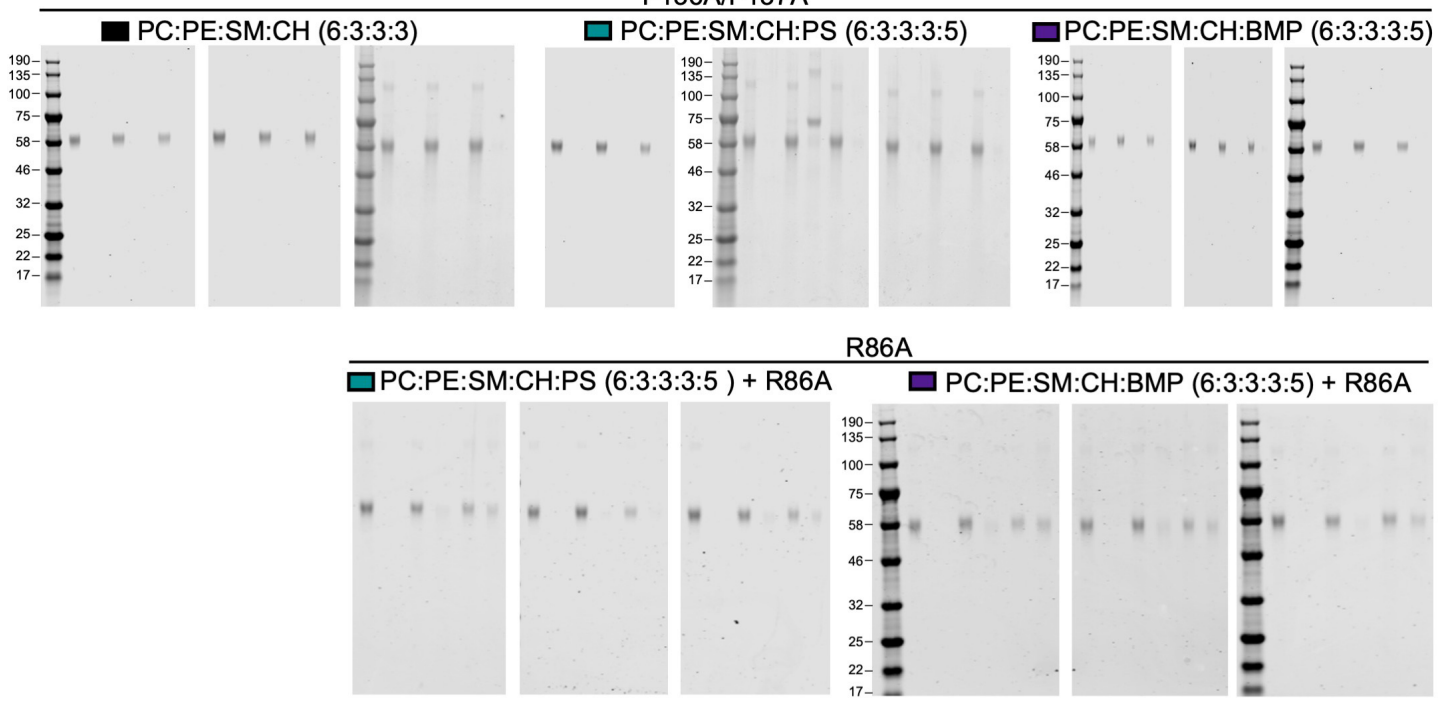

WT
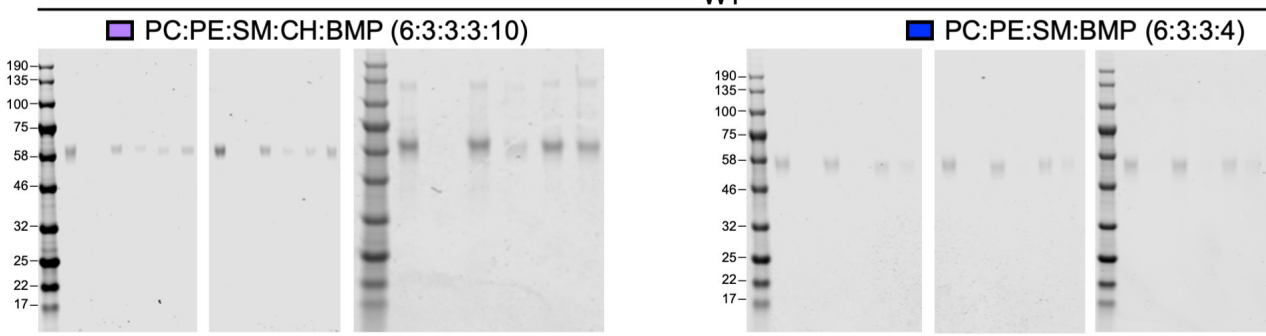

WT
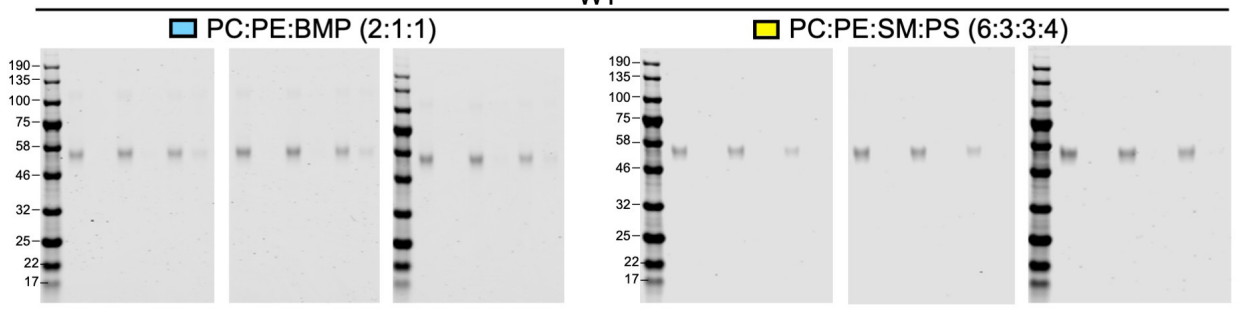

1115

$\square$ PC:PE:SM:CH:PG (6:3:3:3:5)

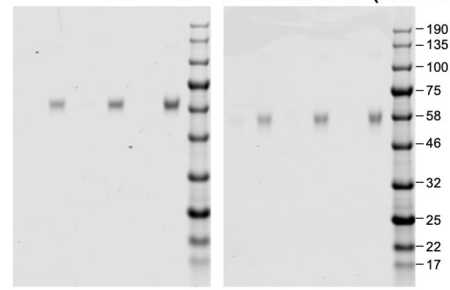

Fig.6D $\alpha-\mathrm{HA}$

Fig. S8. Uncropped gels. Gels shown for all liposome flotation replicates (Fig. 4A), along with 1117 immunoblot from Fig. 6D. 

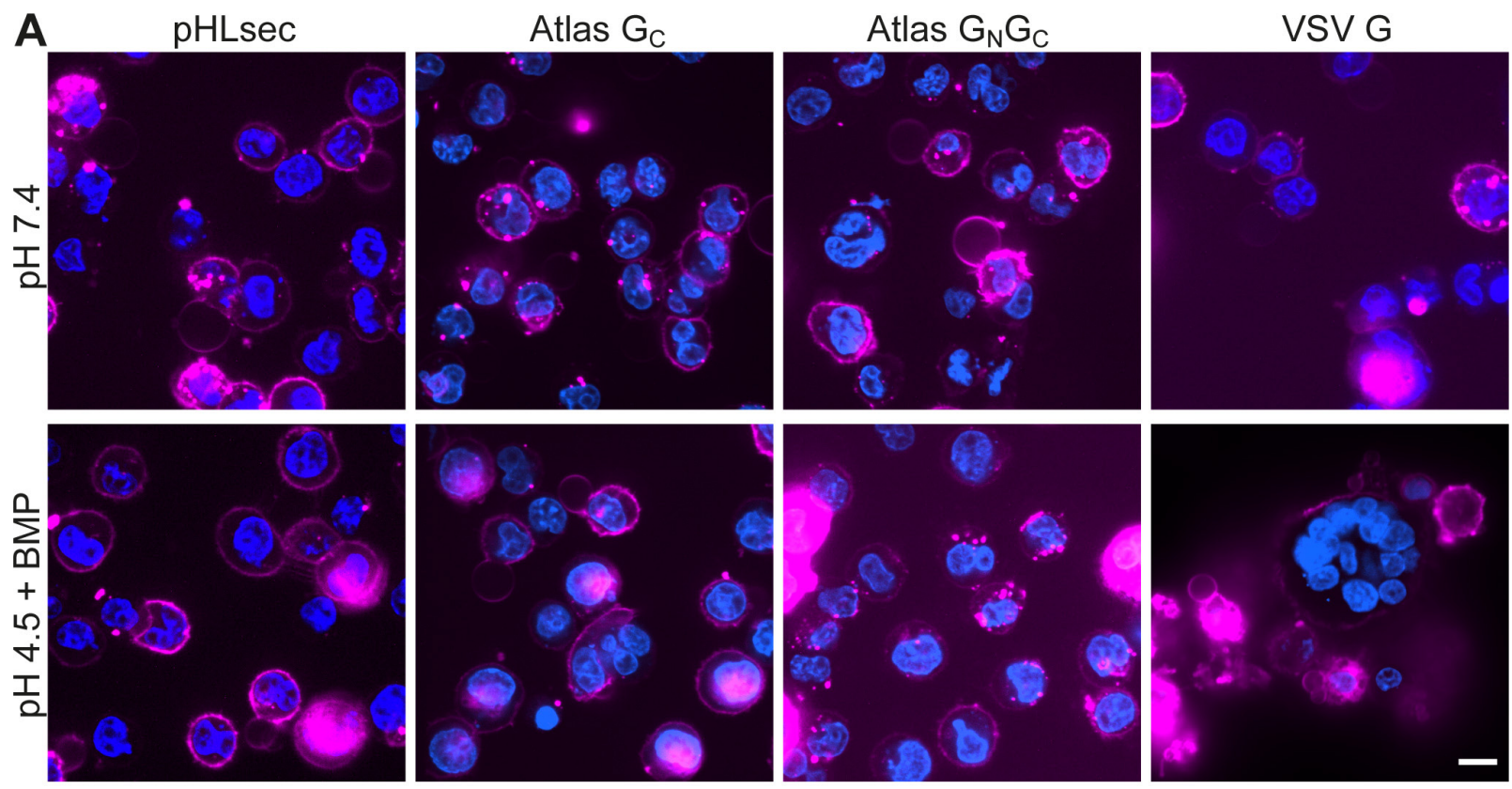

B pHLsec, $\mathrm{pH} 7.4$ (Hoechst, $\alpha-\mathrm{HA}$ )
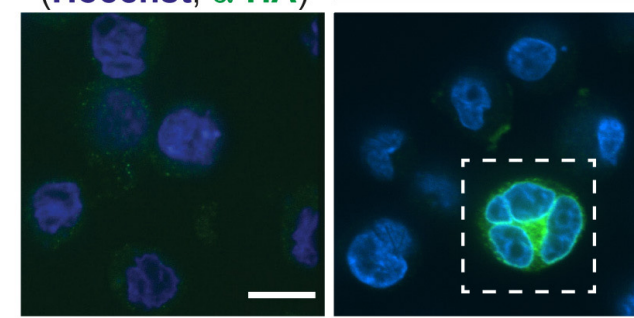

Atlas $\mathrm{G}_{\mathrm{C}}, \mathrm{pH} 4.5+\mathrm{BMP}$ (Hoechst, $\left.\alpha-\mathrm{HA}\right)$

C

pHLsec pH 7.4, Hoechst
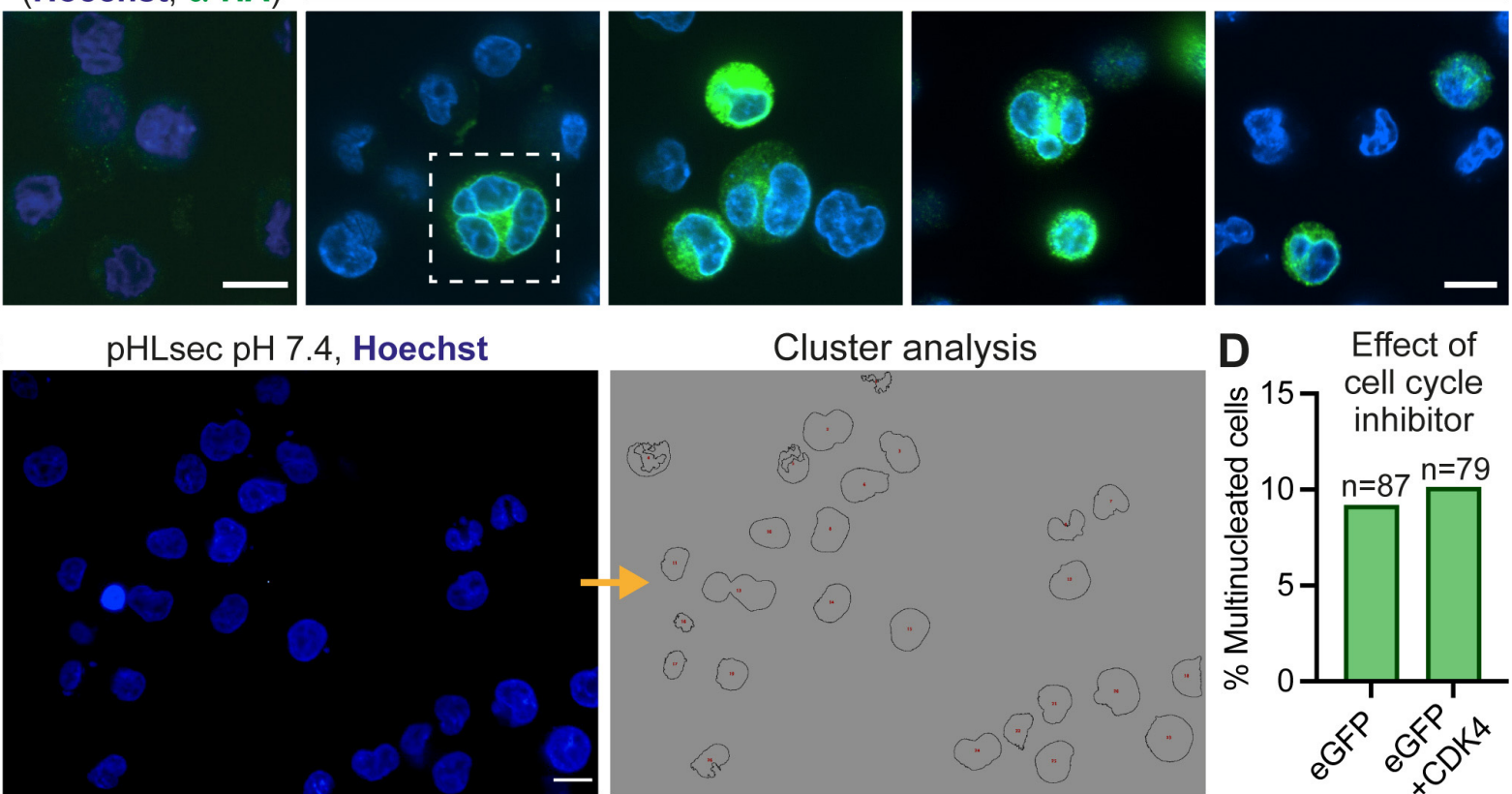

Cluster analysis

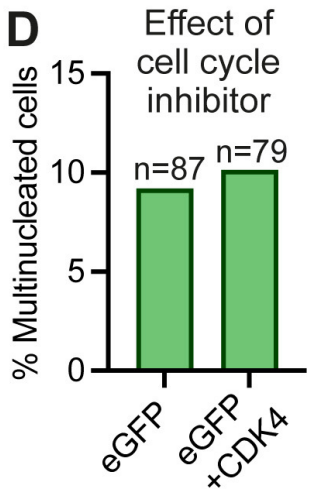

Fig. S9. Cell-cell fusion assay with $\mathrm{CHO}$ cells. (A) Representative confocal micrographs of $\mathrm{CHO}$ cells transfected with plasmid encoding Atlas $\mathrm{G}_{\mathrm{C}}$, Atlas $\mathrm{G}_{\mathrm{N}}-\mathrm{G}_{\mathrm{C}}$, VSV $\mathrm{G}$, or no protein (pHLsec empty vector). Cells were treated with $\mathrm{pH} 4.5$ buffer containing BMP, or $\mathrm{pH} 7.4$ buffer without exogenous lipid. Blue, Hoechst 33342 nuclear stain; Magenta, CellBrite Red plasma membrane dye. (B) Permeabilized and fixed cells transfected with plasmid encoding $\mathrm{G}_{\mathrm{c}}$ stained with anti-HA antibody, to detect the C-terminal HA tag on $\mathrm{G}_{\mathrm{c}}$. Blue, Hoechst 33342; green, antiHA antibody. The boxed region is also shown in Fig. 6B and Movie S1. (C) Left, confocal micrograph of cells transfected with pHLsec plasmid stained with Hoechst 33342. Right, masks from cluster analysis of the Hoechst channel with Fiji (88). All scale bars are $10 \mu \mathrm{m}$. (D) Effect of cell cycle inhibitor CDK4 on the fraction of di- or multinucleated cells. Cell were transfected with a plasmid encoding eGFP (pEGFP-N1) and incubated with $10 \mu \mathrm{M}$ CDK4 (right) or with PBS

1130 (left) for $6 \mathrm{~h}$ at $\mathrm{pH}$ 7.4. $n$, number of nuclei counted. See dataset $\mathbf{S 2}$ for source data. 


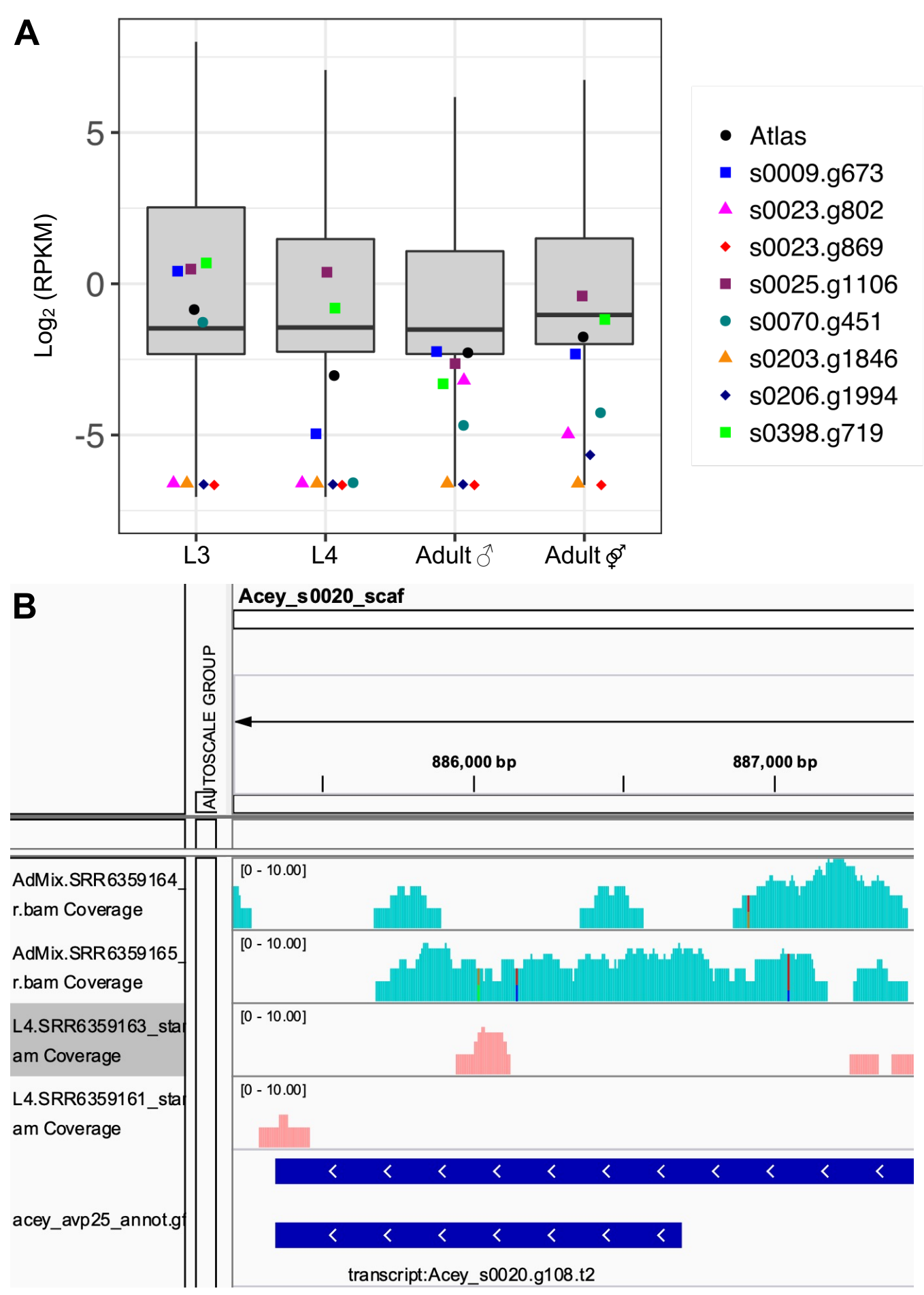

1133 Fig. S10. RNA-Seq analysis of intact $\boldsymbol{A}$. ceylanicum belpaovirus EVEs. (A) Box plots of RNA-Seq expression in Reads Per Kilobase of transcript per Million mapped reads (RPKM) for all annotated genes, and for nine intact $A$. ceylanicum belpaovirus EVEs including Atlas virus, at the L3, L4 and adult (male and mixed sex) developmental stages. RNA-Seq data are from Bernot et al. (71). (B) Integrative Genomics Viewer snapshot showing the distribution of raw RNA-Seq reads over the Atlas virus (Acey_s0020.g108) coding sequence in mixed sex adult worms and L4 larvae with individual unmerged BAM files. 
1141 Table S1. Cryo-EM data collection, structure determination, model building and 1142 refinement parameters and statistics.

Atlas $\mathbf{G}_{\mathrm{c}}$

\begin{tabular}{|c|c|}
\hline Data Collection and Processing & \\
\hline Microscope & FEl Titan Krios \\
\hline Voltage (kV) & 300 \\
\hline Electron exposure (electrons $\AA^{-2}$ ) & 46.12 \\
\hline Exposure per frame (electrons $\AA^{-2}$ ) & 1.28 \\
\hline Defocus range $(\mu \mathrm{m})$ & -1.3 to -3.5 \\
\hline Pixel size $(\AA)$ & 1.047 \\
\hline N. initial particles & 987,570 \\
\hline N. final particles & 197,145 \\
\hline Map resolution $(\AA)$ & 3.76 \\
\hline Map resolution range $(\AA)$ & $3.50-5.20$ \\
\hline FSC threshold for resolution limit & 0.143 \\
\hline Model refinement & \\
\hline Map sharpening $B$ factor $\left(\AA^{2}\right)$ & -180 \\
\hline Symmetry imposed & $\mathrm{C} 3$ \\
\hline Mask correlation coefficient & 0.78 \\
\hline Model composition & \\
\hline N. non-hydrogen atoms & 10,159 \\
\hline Protein residues (chains $A, B, C$ ) & 1,320 \\
\hline R.m.s. deviations & \\
\hline Bond lengths ( $\AA$ ) & 0.005 \\
\hline Bond angles $\left({ }^{\circ}\right)$ & 0.694 \\
\hline Planarity $(\AA)$ & 0.004 \\
\hline B-factors/ADPs* & \\
\hline Minimum & 35 \\
\hline Maximum & 176 \\
\hline Mean & 82 \\
\hline Validation (Phenix 1.15) & \\
\hline MolProbity overall score & 2.23 \\
\hline MolProbity all-atom clashscore & 5.86 \\
\hline Rotamer outliers (\%) & 2.28 \\
\hline Ramachandran plot & \\
\hline$\%$ favored & 86.2 \\
\hline$\%$ allowed & 13.8 \\
\hline$\%$ outliers & 0.0 \\
\hline PDB code & $7 A 4 A$ \\
\hline EMDB code & EMD-11630 \\
\hline EMPIAR code & 10266 \\
\hline
\end{tabular}


1145 Movie S1. Confocal Z-stack of the multinucleated cell shown in Fig. 6B (separate file).

$1146 \mathrm{CHO}$ cell expressing Atlas $\mathrm{G}_{\mathrm{c}}$ following treatment with $\mathrm{pH} 4.5$ and BMP. Blue, Hoechst 33342;

1147 magenta, CellBrite Red; green, a-HA antibody for Atlas $\mathrm{G}_{\mathrm{c}}$ detection. Scale bar, $10 \mu \mathrm{m}$.

1148

1149 Dataset S1. (Separate file)

1150 Source data file for Fig. 4 - liposome binding assays.

1151

1152 Dataset S2. (Separate file)

1153 Source data file for Fig. 6 and Fig. S9 - cell-cell fusion assays.

1154

1155 Dataset S3. (Separate file)

1156 Source data file for Fig. 7 - differential RNA-Seq expression calculations.

1157

1158 Dataset S4. (Separate file)

1159 Source data file for Fig. 7 - original RNA-Seq annotation (GFF) file.

1160

1161 Dataset S5. (Separate file)

1162 RNA-Seq analysis scripts and instructions (also available on Github

1163 [https://github.com/annaprotasio/Merchant_et_al_2020]). 